\title{
Exponential Contraction in Wasserstein Distances for Diffusion Semigroups with Negative Curvature*
}

\author{
Feng-Yu Wang \\ Laboratory of Mathematical and Complex Systems, Beijing Normal University, Beijing 100875, China \\ Department of Mathematics, Swansea University, Singleton Park, SA2 8PP, United Kingdom \\ wangfy@bnu.edu.cn, F.-Y.Wang@swansea.ac.uk
}

September 10, 2019

\begin{abstract}
Let $P_{t}$ be the (Neumann) diffusion semigroup $P_{t}$ generated by a weighted Laplacian on a complete connected Riemannian manifold $M$ without boundary or with a convex boundary. It is well known that the Bakry-Emery curvature is bounded below by a positive constant $\lambda>0$ if and only if

$$
W_{p}\left(\mu_{1} P_{t}, \mu_{2} P_{t}\right) \leq \mathrm{e}^{-\lambda t} W_{p}\left(\mu_{1}, \mu_{2}\right), \quad t \geq 0, p \geq 1
$$

holds for all probability measures $\mu_{1}$ and $\mu_{2}$ on $M$, where $W_{p}$ is the $L^{p}$ Wasserstein distance induced by the Riemannian distance. In this paper, we prove the exponential contraction

$$
W_{p}\left(\mu_{1} P_{t}, \mu_{2} P_{t}\right) \leq c \mathrm{e}^{-\lambda t} W_{p}\left(\mu_{1}, \mu_{2}\right), \quad p \geq 1, t \geq 0
$$

for some constants $c, \lambda>0$ for a class of diffusion semigroups with negative curvature where the constant $c$ is essentially larger than 1. Similar results are derived for SDEs with multiplicative noise by using explicit conditions on the coefficients, which are new even for SDEs with additive noise.
\end{abstract}

AMS subject Classification: 60J75, 47G20, 60G52.

Keywords: Wasserstein distance, diffusion semigroup, Riemannian manifold, curvature condition, SDEs with multiplicative noise.

${ }^{*}$ Supported in part by NNSFC (11131003, 11431014). 


\section{Introduction}

Let $M$ be a $d$-dimensional connected complete Riemannian manifold possibly with a convex boundary $\partial M$. Let $\rho$ be the Riemannian distance. Consider $L=\Delta+Z$ for the LaplaceBeltrami operator $\Delta$ and some $C^{1}$-vector field $Z$ such that the (reflecting) diffusion process generated by $L$ is non-explosive. Then the associated Markov semigroup $P_{t}$ is the (Neumann if $\partial M \neq \emptyset$ ) semigroup generated by $L$ on $M$. In particular, it is the case when the curvature of $L$ is bounded below; that is,

$$
\operatorname{Ric}_{Z}:=\operatorname{Ric}-\nabla Z \geq K
$$

holds for some constant $K \in \mathbb{R}$. Here and throughout the paper, we write $\mathscr{T} \geq h$ for a (not necessarily symmetric) 2-tensor $\mathscr{T}$ and a function $h$ provided

$$
\mathscr{T}(X, X) \geq h(x)|X|^{2}, \quad X \in T_{x} M, x \in M .
$$

There exist many inequalities on $P_{t}$ which are equivalent to the curvature condition (1.1), see $[6,20,23,42]$ for details. In particular, for any constant $K \in \mathbb{R}$, the Wasserstein distance inequality

$$
W_{p}\left(\mu_{1} P_{t}, \mu_{2} P_{t}\right) \leq \mathrm{e}^{-K t} W_{p}\left(\mu_{1}, \mu_{2}\right), \quad t \geq 0, p \geq 1, \mu_{1}, \mu_{2} \in \mathscr{P}(M)
$$

is equivalent to the curvature condition (1.1). Here, $\mathscr{P}(M)$ is the class of all probability measures on $M ; W_{p}$ is the $L^{p}$-Warsserstein distance induced by $\rho$, i.e.,

$$
W_{p}\left(\mu_{1}, \mu_{2}\right):=\inf _{\pi \in \mathscr{C}\left(\mu_{1}, \mu_{2}\right)}\|\rho\|_{L^{p}(\pi)}, \quad \mu_{1}, \mu_{2} \in \mathscr{P}(M),
$$

where $\mathscr{C}\left(\mu_{1}, \mu_{2}\right)$ is the class of all couplings of $\mu_{1}$ and $\mu_{2}$; and for a Markov operator $P$ on $\mathscr{B}_{b}(M)$ (i.e. $P$ is a positivity-preserving linear operator with $P 1=1$ ),

$$
(\nu P)(A):=\nu\left(P 1_{A}\right), \quad A \in \mathscr{B}(M), \nu \in \mathscr{P}(M),
$$

where $\nu(f):=\int_{M} f \mathrm{~d} \nu$ for $f \in L^{1}(\nu)$. In some references, $\nu P$ is also denoted by $P^{*} \nu$. In the sequel we will use $P_{t}^{*}$ to stand for the adjoint operator of $P_{t}$ in $L^{2}(\mu)$ for the invariant probability measure $\mu$, hence adopt the notation $\nu P$ rather than $P^{*} \nu$ to avoid confusion. When the curvature is positive (i.e. $K>0$ ), (1.2) implies the $W_{p}$-exponential contraction of $P_{t}$ for $p \geq 1$.

In this paper, we aim to consider the case when (1.1) only holds for some negative constant $K$, and to prove the exponential contraction

$$
W_{p}\left(\mu_{1} P_{t}, \mu_{2} P_{t}\right) \leq c \mathrm{e}^{-\lambda t} W_{p}\left(\mu_{1}, \mu_{2}\right), \quad t \geq 0, p \geq 1, \mu_{1}, \mu_{2} \in \mathscr{P}(M)
$$

for some constants $c, \lambda>0$. It is crucial that the exponential rate $\lambda$ is independent of $p$. Due to the equivalence of (1.1) and (1.2), in the negative curvature case it is essential that $c>1$. 
According to [37], even when $\operatorname{Ric}_{Z}$ is unbounded below, i.e. $\operatorname{Ric}_{Z}$ goes to $-\infty$ when $\rho_{o}:=\rho(o, \cdot) \rightarrow \infty$ for a fixed $o \in M$, there may exist the log-Sobolev inequality which implies the exponentially convergence of $P_{t}$ in entropy. This suggests that (1.3) may also hold for a class of diffusion semigroups with negative curvature.

Recently, some efforts have been made in this direction for $M=\mathbb{R}^{d}$, see $[11,12,18]$. More precisely, let $P_{t}$ be the diffusion semigroup for the solution to the following SDE on $\mathbb{R}^{d}$ :

$$
\mathrm{d} X_{t}=\sqrt{2} \mathrm{~d} B_{t}+b\left(X_{t}\right) \mathrm{d} t,
$$

where $B_{t}$ is the $d$-dimensional Brownian motion and $b: \mathbb{R}^{d} \rightarrow \mathbb{R}^{d}$ is continuous. If there exist constants $K_{1}, K_{2}, r_{0}>0$ such that

$$
\langle b(x)-b(y), x-y\rangle \leq 1_{|x-y| \leq r_{0}}\left(K_{1}+K_{2}\right)|x-y|^{2}-K_{2}|x-y|^{2}, \quad x, y \in \mathbb{R}^{d},
$$

then due to $[11,12]$ we have

$$
W_{1}\left(\delta_{x} P_{t}, \delta_{y} P_{t}\right) \leq c \mathrm{e}^{-\lambda t}|x-y|, \quad x, y \in \mathbb{R}^{d}, t \geq 0
$$

for some constants $c, \lambda>0$, where $\delta_{x}$ is the Dirac measure at point $x$. Indeed, $[11,12]$ proved the $W_{1}$-exponential contraction with respect to a modified distance $f(|x-y|)$ in place of $|x-y|$ as constructed in [8,9] for estimates of the spectral gap using the coupling by reflection. Under condition (1.4) the modified distance is comparable with the usual one so that (1.5) follows. As mentioned in [12] that there is essential difficulty to prove (1.3) for $p>1$ even for this flat case.

In Luo and Wang [18] the estimate (1.5) was extended as

$$
W_{p}\left(\delta_{x} P_{t}, \delta_{y} P_{t}\right) \leq c \mathrm{e}^{-\lambda t / p}\left(|x-y|+|x-y|^{\frac{1}{p}}\right), \quad x, y \in \mathbb{R}^{d}, t \geq 0, p \geq 1
$$

for some constants $c, \lambda>0$. Comparing with (1.3) which is equivalent to

$$
W_{p}\left(\delta_{x} P_{t}, \delta_{y} P_{t}\right) \leq c \mathrm{e}^{-\lambda t}|x-y|, \quad p \geq 1, x, y \in \mathbb{R}^{d}, t \geq 0
$$

according to [17] (see Proposition 3.1 below), (1.6) is less sharp for small $|x-y|$ and/or large $p$. It is open whether (1.4), or in the Riemannian setting that $\mathrm{Ric}_{Z}$ is uniformly positive outside a compact domain, implies (1.3) for some constants $c, \lambda>0$.

As in $[16,17]$, we will consider the Warsserstein distances induced by Young functions in the class

$$
\begin{array}{r}
\mathscr{N}:=\left\{\Phi \in C^{1}([0, \infty) ;[0, \infty)): \Phi^{\prime}\right. \text { is nonnegative and increasing, } \\
\left.\Phi(0)=0, \Phi(r)>0 \text { for } r>0, \lim _{r \rightarrow \infty} \frac{\Phi(r)}{r}=\infty\right\} .
\end{array}
$$

For any $\Phi \in \mathscr{N}$ and a measure $\nu$ on $M$, consider the gauge norm in $L^{\Phi}(\nu)$ :

$$
\|f\|_{L^{\Phi}(\nu)}:=\inf \left\{r>0: \nu\left(\Phi\left(r^{-1}|f|\right)\right) \leq 1\right\}, \quad \inf \emptyset:=\infty .
$$


In particular, we have $\|f\|_{L^{\Phi^{p}(\nu)}}=\|f\|_{L^{p}(\nu)}$ for $\Phi_{p}(r):=r^{p}, p \in(1, \infty)$. This is the reason why we do not take $\Phi_{p}(r)=\frac{1}{p} r^{p}$ in the characterization of Legendre conjugates. We extend the notion $\Phi_{p}$ to $p=1, \infty$ by letting $\Phi_{1}(r)=r, \Phi_{\infty}=\lim _{p \rightarrow \infty} \Phi_{p}$ and $\|f\|_{L^{\Phi_{p}(\nu)}}=\|f\|_{L^{p}(\nu)}$ for all $p \in[1, \infty]$. Now, let

$$
W_{\Phi}\left(\mu_{1}, \mu_{2}\right)=\inf _{\pi \in \mathscr{C}\left(\mu_{1}, \mu_{2}\right)}\|\rho\|_{L^{\Phi}(\pi)}, \quad \Phi \in \overline{\mathscr{N}}:=\mathscr{N} \cup\left\{\Phi_{1}, \Phi_{\infty}\right\} .
$$

In particular, $W_{\Phi_{p}}=W_{p}$ for $p \in[1, \infty]$. We aim to prove the exponential decay

$$
W_{\Phi}\left(\delta_{x} P_{t}, \delta_{y} P_{t}\right) \leq \frac{c}{\Phi^{-1}(1)} \mathrm{e}^{-\lambda t} \rho(x, y), \quad x, y \in M, t \geq 0, \Phi \in \overline{\mathscr{N}}
$$

when (1.1) only holds for a negative constant $K$, where $\Phi^{-1}$ is the inverse of $\Phi\left(\neq \Phi_{\infty}\right)$ and we set $\Phi_{\infty}^{-1}(1)=1$ by convention.

To extend condition (1.4) to the Riemannian setting, consider the index

$$
I(x, y)=\int_{0}^{\rho(x, y)} \sum_{i=1}^{d-1}\left\{\left|\nabla_{\dot{\gamma}} J_{i}\right|^{2}-\left\langle\mathscr{R}\left(\dot{\gamma}, J_{i}\right) \dot{\gamma}, J_{i}\right\rangle\right\}\left(\gamma_{s}\right) \mathrm{d} s, \quad x, y \in M,
$$

where $\rho$ is the Riemannian distance, $\mathscr{R}$ is the curvature tensor; $\gamma:[0, \rho(x, y)] \rightarrow M$ is the minimal geodesic from $x$ to $y$ with unit speed; $\left\{J_{i}\right\}_{i=1}^{d-1}$ are Jacobi fields along $\gamma$ such that

$$
J_{i}(y)=P_{x, y} J_{i}(x), \quad i=1, \ldots, d-1
$$

holds for the parallel transform $P_{x, y}: T_{x} M \rightarrow T_{y} M$ along the geodesic $\gamma$, and $\left\{\dot{\gamma}(s), J_{i}(s)\right.$ : $1 \leq i \leq d-1\}(s=0, \rho(x, y))$ is an orthonormal basis of the tangent space (at points $x$ and $y$, respectively).

Note that when $(x, y) \in \operatorname{Cut}(M)$, i.e. $x$ is in the cut-locus of $y$, the minimal geodesic may be not unique. As a convention in the literature, all conditions on the index $I$ are given outside $\operatorname{Cut}(M)$. We now extend condition (1.4) to the non-flat case as follows: for some constants $K_{1}, K_{2}>0$

$$
\begin{aligned}
I_{Z}(x, y) & :=I(x, y)+\langle Z, \nabla \rho(\cdot, y)\rangle(x)+\langle Z, \nabla \rho(x, \cdot,)\rangle(y) \\
& \leq\left\{\left(K_{1}+K_{2}\right) 1_{\left\{\rho(x, y) \leq r_{0}\right\}}-K_{2}\right\} \rho(x, y), \quad x, y \in M .
\end{aligned}
$$

In the flat case we have $I(x, y)=0$ and $\rho(x, y)=|x-y|$, so that this condition reduces back to (1.4). Moreover, the curvature condition (1.1) is equivalent to

$$
I_{Z}(x, y) \leq-K \rho(x, y), \quad x, y \in M
$$

so that (1.8) implies $\operatorname{Ric}_{Z} \geq-\left(K_{1}+K_{2}\right)$.

In the next section, we state our main results and present examples. With condition (1.8) we first extend the main results of $[11,18]$ to the present Riemannian setting and give the exponential convergence of $P_{t}$ in $W_{2}$. Under the ultracontractivity and condition (1.1) for some $K<0$, our the second result ensures the desired inequality (1.7). Finally, we extend these results to SDEs with multiplicative noise by using explicit conditions on the coefficients. To prove these results, we make some preparations in Section 3. Complete proofs of the main results are addressed in Sections 4-6 respectively. 


\section{Main Results and examples}

We first consider the Riemannian setting, then extend to SDEs with multiplicative noise by using explicit conditions on the coefficients instead of the less explicit curvature condition.

\subsection{The Riemannian setting}

We start with condition (1.8). Besides the extension of (1.6), this condition also implies the hypercontractivity and the exponential convergence in $W_{2}$ for the semigroup $P_{t}$. For a measure $\mu$ and constants $p, q \geq 1$, let $\|\cdot\|_{L^{p}(\mu) \rightarrow L^{q}(\mu)}$ stand for the operator norm form $L^{p}(\mu)$ to $L^{q}(\mu)$. Recall that $P_{t}$ is called hypercontractive if it has a unique invariant probability measure $\mu$ and $\left\|P_{t}\right\|_{L^{2}(\mu) \rightarrow L^{4}(\mu)}=1$ holds for large $t>0$. By interpolation theorem, $\left\|P_{t}\right\|_{L^{2}(\mu) \rightarrow L^{4}(\mu)}=1$ can be replaced by $\left\|P_{t}\right\|_{L^{p}(\mu) \rightarrow L^{q}(\mu)}=1$ for some $\infty>q>p>1$. Let $\rho_{o}=(o, \cdot)$ for a fixed point $o \in M$.

Theorem 2.1. Let (1.8) hold for some constants $K_{1}, K_{2}$ and $r_{0}>0$. Then:

(1) There exist two constants $c, \lambda>0$ such that for any $\Phi \in \overline{\mathscr{N}}$ and $x, y \in M$,

$$
W_{\Phi}\left(\delta_{x} P_{t}, \delta_{y} P_{t}\right) \leq \inf \left\{r>0: \sup _{s \in\left(0,1+r_{0}+\rho(x, y)\right]} \frac{\Phi\left(r^{-1} s\right)}{s} \leq \frac{\mathrm{e}^{\lambda t}}{c \rho(x, y)}\right\}, \quad t \geq 0 .
$$

In particular, there exist constants $c, \lambda>0$ such that

$$
W_{p}\left(\delta_{x} P_{t}, \delta_{y} P_{t}\right) \leq\left\{c \mathrm{e}^{-\lambda t}\right\}^{\frac{1}{p}}\left(\rho(x, y)+\rho(x, y)^{\frac{1}{p}}\right), \quad p \geq 1, t \geq 0, x, y \in M .
$$

(2) If $P_{t}$ has an invariant probability measure $\mu$ with $\mu\left(\mathrm{e}^{\varepsilon \rho_{0}^{2}}\right)<\infty$ for some constant $\varepsilon>0$, then the log-Sobolev inequality

$$
\mu\left(f^{2} \log f^{2}\right) \leq C \mu\left(|\nabla f|^{2}\right)+\mu\left(f^{2}\right) \log \mu\left(f^{2}\right), \quad f \in C_{b}^{1}(M)
$$

holds for some constant $C>0$. Consequently, $P_{t}$ is hypercontractive. If moreover $\partial M=\emptyset$, then exist constants $c, \lambda>0$ such that

$$
W_{2}\left(\nu P_{t}, \mu\right) \leq c \mathrm{e}^{-\lambda t} W_{2}(\nu, \mu), \quad t \geq 0, \nu \in \mathscr{P}(M) .
$$

To illustrate this result, we present below a consequence with explicit conditions on Ric and $\nabla Z$ for which $\operatorname{Ric}_{Z}$ may be negative everywhere.

Corollary 2.2. If there exist constants $\delta_{1}, \delta_{2}>0$ such that $\mathrm{Ric} \geq \delta_{1}$ and

$$
\nabla Z \leq-\delta_{2} \text { outside a compact set, }
$$

then Theorem 2.1(1) holds, and $P_{t}$ has a unique invariant probability measure $\mu$ such that (3.4) and (2.3) hold for some constants $C, c, \lambda>0$. 
Next, we introduce sufficient conditions for (1.7) to hold, these conditions also allow $\mathrm{Ric}_{Z}$ to be negative. Due to technical reason, we will need the ultracontractivity of $P_{t}$, which is essentially stronger than the hypercontractivity. We call $P_{t}$ ultracontractive if $\left\|P_{t}\right\|_{L^{1}(\mu) \rightarrow L^{\infty}(\mu)}<\infty$ for all $t>0$. The ultracontractivity implies that $P_{t}$ has a density $p_{t}(x, y)$ with respect to $\mu$ (called heat kernel) and

$$
\left\|p_{t}\right\|_{L^{\infty}(\mu \times \mu)}=\left\|P_{t}\right\|_{L^{1}(\mu) \rightarrow L^{\infty}(\mu)}<\infty, \quad t>0 .
$$

In references (see e.g. [10]), the ultracontractivity is also defined by $\left\|P_{t}\right\|_{L^{2}(\mu) \rightarrow L^{\infty}(\mu)}<\infty$ for $t>0$. When $P_{t}$ is symmetric in $L^{2}(\mu)$ we have

$$
\left\|P_{t}\right\|_{L^{1}(\mu) \rightarrow L^{\infty}(\mu)} \leq\left\|P_{t / 2}\right\|_{L^{2}(\mu) \rightarrow L^{\infty}(\mu)}^{2}, \quad t>0,
$$

so that these two definitions are equivalent. However, when $P_{t}$ is non-symmetric, the former might be stronger than the latter. The appearance of the ultracontractivity in our study is very nature: by Theorem 2.3(1) we already have (1.7) for $\Phi=\Phi_{1}$ (the weakest case), and by the ultracontractivity we are able to deduce the inequality from $\Phi_{1}$ to $\Phi_{\infty}$ (the strongest case). On the other hand, the result also indicates that (1.7) implies the hypercontractivity of $P_{t}$.

Theorem 2.3. Assume that $\mathrm{Ric}_{Z}$ is bounded below.

(1) If $P_{t}$ is ultracontractive, then there exist constants $c, \lambda>0$ such that for any $\Phi \in \overline{\mathscr{N}}$,

$$
W_{\Phi}\left(\delta_{x} P_{t}, \delta_{y} P_{t}\right) \leq \frac{c}{\Phi^{-1}(1)} \mathrm{e}^{-\lambda t} \min \left\{\rho(x, y), G_{\Phi}(t)\right\}, \quad t>0, x, y \in M
$$

holds for

$$
G_{\Phi}(t):=\inf \left\{r>0:(\mu \times \mu)\left(\Phi\left(r^{-1} \rho\right)\right) \leq\left\|P_{t / 2}\right\|_{L^{1}(\mu) \rightarrow L^{\infty}(\mu)}^{-2}\right\} .
$$

Consequently, for any $p \in[1, \infty], t \geq 0$ and $\mu_{1}, \mu_{2} \in \mathscr{P}(M)$,

$$
W_{p}\left(\mu_{1} P_{t}, \mu_{2} P_{t}\right) \leq c \mathrm{e}^{-\lambda t} \min \left\{W_{p}\left(\mu_{1}, \mu_{2}\right),\|\rho\|_{L^{p}(\mu \times \mu)}\left\|P_{t / 2}\right\|_{L^{1}(\mu) \rightarrow L^{\infty}(\mu)}^{\frac{2}{p}}\right\} .
$$

(2) On the other hand, if there exist constants $c, \lambda>0$ such that

$$
W_{\infty}\left(\delta_{x} P_{t}, \delta_{y} P_{t}\right) \leq c \mathrm{e}^{-\lambda t} \rho(x, y), \quad x, y \in M, t \geq 0,
$$

then the log-Sobolev inequality (3.4) holds for $c=\frac{2 c^{2}}{\lambda}$, so that $P_{t}$ is hypercontractive.

We note that in Theorem 2.3(1) we have $\|\rho\|_{L^{p}(\mu \times \mu)}<\infty$ for $p \in[1, \infty)$. Indeed, since $\operatorname{Ric}_{Z}$ is bounded below, by [25, Theorem 2.1] the ultracontractivity implies the super logSobolev inequality (3.3) below, so that due to Herbst we have $(\mu \times \mu)\left(\mathrm{e}^{r \rho^{2}}\right)<\infty$ for all $r>0$ (see e.g. $[1,2]$ ). Therefore, $G_{\Phi}(t)<\infty$ for $t>0$ and $\Phi \in \mathscr{N}$ satisfying

$$
\limsup _{r \rightarrow \infty} \frac{\log \Phi(r)}{r^{2}}<\infty \text {. }
$$


In the symmetric case (i.e. $Z=\nabla V$ for some $V \in C^{2}(M)$ ), explicit sufficient conditions for the ultracontractivity have been introduced in [37] by using the dimension-free Harnack inequality in the sense of [33]. Together with a suitable exponential estimate on the diffusion process, this inequality implies $\left\|P_{t}\right\|_{L^{2}(\mu) \rightarrow L^{\infty}(\mu)}<\infty$ for $t>0$ and thus, $P_{t}$ is ultracontractive due to (2.5). The conditions can be formulated as

$$
-\nabla Z \geq \Psi_{1} \circ \rho_{o} \text { and Ric } \geq-\Psi_{2} \circ \rho_{o} \text { hold outside a compact subset of } M,
$$

where $\Psi_{1}, \Psi_{2}:(0, \infty) \rightarrow(0, \infty)$ are increasing functions such that

$$
\int_{1}^{\infty} \frac{\mathrm{d} s}{\sqrt{s} \int_{0}^{\sqrt{s}} \Psi_{1}(u) \mathrm{d} u}<\infty, \quad \lim _{r \rightarrow \infty} \min \left\{\Psi_{1}(r), \frac{\left(\int_{0}^{r} \Psi_{1}(s) \mathrm{d} s\right)^{2}}{\Psi_{1}(r)}\right\}=\infty
$$

and for some constants $\theta \in(0,1 /(1+\sqrt{2}))$ and $C>0$,

$$
\sqrt{\Psi_{2}(r+t)(d-1)} \leq \theta \int_{0}^{r} \Psi_{1}(s) \mathrm{d} s+\frac{1}{2} \int_{0}^{t / 2} \Psi_{1}(s) \mathrm{d} s+C, \quad r, t \geq 0 .
$$

When Ric is bounded below, (2.12) as well as the second inequality in (2.10) hold for $\Psi_{2}$ being a large enough constant. In general, since $\int_{0}^{r} \Psi_{1}(s) \mathrm{d} s \geq 2 \int_{0}^{r / 2} \Psi_{1}(s) \mathrm{d} s$, (2.12) with $\theta=\frac{1}{4}<\frac{1}{1+\sqrt{2}}$ follows from

$$
\begin{aligned}
\sqrt{\Psi_{2}(r)(d-1)} & \leq \frac{1}{2} \inf _{t \in[0, r]}\left\{\int_{0}^{t / 2} \Psi_{1}(s) \mathrm{d} s+\int_{0}^{(r-t) / 2} \Psi_{1}(s) \mathrm{d} s\right\}+C \\
& =\int_{0}^{r / 4} \Psi_{1}(s) \mathrm{d} s+C, \quad r \geq 0 .
\end{aligned}
$$

Since (2.5) fails for non-symmetric semigroups, we apply the inequality

$$
\left\|P_{t}\right\|_{L^{1}(\mu) \rightarrow L^{\infty}(\mu)} \leq\left\|P_{t / 2}\right\|_{L^{1}(\mu) \rightarrow L^{2}(\mu)}\left\|P_{t / 2}\right\|_{L^{2}(\mu) \rightarrow L^{\infty}(\mu)}
$$

due to the semigroup property. So, to ensure the ultracontractivity, we need an additional condition implying $\left\|P_{t}\right\|_{L^{1}(\mu) \rightarrow L^{2}(\mu)}<\infty$ (see Corollary 2.4(2) below).

To estimate $G_{\Phi}(t)$ in $(2.6)$ using $\Psi_{1}$, we introduce

$$
\Lambda_{1}(r):=\frac{1}{\sqrt{r}} \int_{0}^{\sqrt{r}} \Psi_{1}(s) \mathrm{d} s, \quad \Lambda_{2}(r):=\int_{r}^{\infty} \frac{\mathrm{d} s}{\sqrt{s} \int_{0}^{\sqrt{s}} \Psi_{1}(u) \mathrm{d} u}, \quad r>0 .
$$

Obviously, the inverse function $\Lambda_{2}^{-1}$ exists on $(0, \infty)$, and since $\Lambda_{1}$ is increasing with $\Lambda_{1}(\infty)=$ $\infty$, we have

$$
\Lambda_{1}^{-1}(r):=\inf \left\{s \geq 0: \Lambda_{1}(s) \geq r\right\}<\infty, \quad r \geq 0 .
$$

Corollary 2.4. Assume that (2.11) and (2.12) hold for some constants $\theta \in(0,1 /(1+\sqrt{2}))$ and $C>0$. 
(1) If $P_{t}$ is symmetric, i.e. $Z=\nabla V$ for some $V \in C^{2}(M)$, then there exist constants $c, \lambda>0$ such that (2.6) and (2.8) hold for

$$
G_{\Phi}(t):=\inf \left\{r>0:(\mu \times \mu)\left(\Phi\left(r^{-1} \rho\right)\right) \leq \mathrm{e}^{-c-c t^{-1}\left\{1+\Lambda_{1}^{-1}\left(c t^{-1}\right)-\Lambda_{2}^{-1}\left(c^{-1} t\right)\right\}}\right\}, \quad t>0 .
$$

(2) If $P_{t}$ is non-symmetric but there exists continuous $h \in C([0,1] ;[0, \infty))$ with $h(r)>0$ for $r>0$ such that $\int_{0}^{1} \frac{h(r)}{r} \mathrm{~d} r<\infty$ and

$$
H(\theta):=\int_{0}^{1} \frac{\theta}{h(r)}\left\{1+\Lambda_{1}^{-1}(\theta / h(r))+\Lambda_{2}^{-1}(h(r) / \theta)\right\} \mathrm{d} r<\infty, \quad \theta>0,
$$

then there exist constants $c, \lambda>0$ such that (2.6) holds for

$$
G_{\Phi}(t):=\inf \left\{\lambda>0:(\mu \times \mu)\left(\Phi\left(\lambda^{-1} \rho\right)\right) \leq \mathrm{e}^{-c-c t^{-1}\left\{1+\Lambda_{1}^{-1}\left(c t^{-1}\right)-\Lambda_{2}^{-1}\left(c^{-1} t\right)\right\}-c H\left(c t^{-1}\right)}\right\} .
$$

To conclude this part, we present a simple example to illustrate Corollary 2.4.

Example 2.1. Let $M$ have non-positive sectional curvatures and a pole $o \in M$. Let $Z=Z_{0}-\delta \nabla \rho_{o}^{2+\varepsilon}$ outside a compact domain, where $\delta, \varepsilon>0$ are constants and $Z_{0}$ is a $C^{1}$ vector field with

$$
\limsup _{\rho_{o} \rightarrow \infty} \frac{\left|\nabla Z_{0}\right|}{\rho_{o}^{\varepsilon}}<\delta(1+\varepsilon)(2+\varepsilon) .
$$

Let $\Psi_{2}:(0, \infty) \rightarrow(0, \infty)$ be increasing such that

$$
\text { Ric } \geq-\Psi_{2}\left(\rho_{o}\right), \lim _{r \rightarrow \infty} \frac{\Psi_{2}(r)}{r^{2(1+\varepsilon)}}=0 .
$$

By (2.15), (2.16) and the Hessian comparison theorem, we see that (2.10), (2.11) and (2.13) hold with $\Psi_{1}(r)=c_{1} r^{\varepsilon}$ for some constant $c_{1}>0$. By (2.14), there exists a constant $C>0$ such that

$$
\Lambda_{1}^{-1}(\theta / h(r))+\Lambda^{-1}(h(r) / \theta) \leq C \theta^{\frac{2}{\varepsilon}} h(r)^{-\frac{2}{v} v} .
$$

Taking, for instance, $h(r)=r^{\frac{\varepsilon}{4}}$ in Corollary 2.4(2), we may find out constants $c, \lambda>0$ such that for any $p \geq 1$,

$$
W_{p}\left(\mu_{1} P_{t}, \mu_{2} P_{t}\right) \leq c \mathrm{e}^{-\lambda t} \min \left\{W_{p}\left(\mu_{1}, \mu_{2}\right),\|\rho\|_{L_{p}(\mu \times \mu)} \exp \left[\frac{c}{p t^{1+\frac{2}{\varepsilon}}}\right]\right\}, \quad t>0, \mu_{1}, \mu_{2} \in \mathscr{P}(M) .
$$

\subsection{SDEs with multiplicative noise}

Consider the following SDE on $\mathbb{R}^{d}$ :

$$
\mathrm{d} X_{t}=b\left(X_{t}\right) \mathrm{d} t+\sqrt{2} \sigma\left(X_{t}\right) \mathrm{d} B_{t},
$$


where $B_{t}$ is the $m$-dimensional Brownian motion, $b: \mathbb{R}^{d} \rightarrow \mathbb{R}^{d}$ and $\sigma: \mathbb{R}^{d} \rightarrow \mathbb{R}^{d} \otimes \mathbb{R}^{m}$ (the space of $d \times m$-matrices) are locally Lipshitz such that

$$
\|\sigma\|_{H S}^{2}(x)+\langle b(x), x\rangle \leq C\left(1+|x|^{2}\right), \quad x \in \mathbb{R}^{d}
$$

holds for some constant $C>0$, where and in the following, $\|\cdot\|_{H S}$ and $\|\cdot\|$ denote the Hilbert-Schmidt and the operator norms respectively. Then the SDE has a unique solution $\left\{X_{t}(x)\right\}_{t \geq 0}$ for every initial point $x \in \mathbb{R}^{d}$. Let $P_{t}$ be the associated Markov semigroup:

$$
P_{t} f(x):=\mathbb{E}\left[f\left(X_{t}(x)\right)\right], \quad t \geq 0, x \in \mathbb{R}^{d}, f \in \mathscr{B}_{b}\left(\mathbb{R}^{d}\right) .
$$

We intend to investigate the $W_{p^{-}}$exponential contraction for $p \in[1, \infty)$. As mentioned in Introduction that existing results only apply to $p=1$ and $\sigma=I$, and as mentioned in $[12,18]$ that there is essential difficulty to prove (1.3) for $p>1$ even for $\sigma=I$. So, the present study is non-trivial.

Corresponding to that (1.1) implies (1.2) in the Riemannian setting, we have the following assertion.

Theorem 2.5. Let $p \in[1, \infty)$. If

$$
\begin{aligned}
& \frac{(p-2)\left|(\sigma(x)-\sigma(y))^{*}(x-y)\right|^{2}}{|x-y|^{2}}+\|\sigma(x)-\sigma(y)\|_{H S}^{2}+\langle b(x)-b(y), x-y\rangle \\
& \leq-K_{p}|x-y|^{2}, \quad x \neq y \in \mathbb{R}^{d}
\end{aligned}
$$

holds for some constant $K_{p} \in \mathbb{R}$, then

$$
W_{p}\left(\mu_{1} P_{t}, \mu_{2} P_{t}\right) \leq \mathrm{e}^{-K_{p} t} W_{p}\left(\mu_{1}, \mu_{2}\right), \quad t \geq 0, \mu_{1}, \mu_{2} \in \mathscr{P}\left(\mathbb{R}^{d}\right) .
$$

Note that this result does apply to $p=\infty$ when $\sigma$ is non-constant. Next, as in the Riemannian case, we intend to prove the exponential contraction in $W_{p}$ when (2.18) only holds for some negative constant $K_{p}$. To this end, we need the SDE to be non-degenerate. The following result contains analogous assertions in Theorems 2.1 and 2.3, where the first assertion extends (1.5) to the multiplicative noise setting.

Theorem 2.6. Assume that $\lambda_{0}^{-2} I \geq \sigma \sigma^{*} \geq \lambda_{0}^{2} I$ for some constant $\lambda_{0} \in(0,1)$.

(1) If there exist constants $K_{1}, K_{2}, r_{0}>0$ such that $Z$ and $\sigma_{0}:=\sqrt{\sigma \sigma^{*}-\lambda_{0}^{2} I}$ satisfy

$$
\begin{aligned}
& \left\|\sigma_{0}(x)-\sigma_{0}(y)\right\|_{H S}^{2}-\frac{\left|(\sigma(x)-\sigma(y))^{*}(x-y)\right|^{2}}{|x-y|^{2}}+\langle b(x)-b(y), x-y\rangle \\
& \leq\left\{\left(K_{1}+K_{2}\right) 1_{\left\{|x-y| \leq r_{0}\right\}}-K_{2}\right\}|x-y|^{2}, \quad x, y \in \mathbb{R}^{d},
\end{aligned}
$$

then there exist constants $c, \lambda>0$ such that

$$
W_{1}\left(\mu_{1} P_{t}, \mu_{2} P_{t}\right) \leq c \mathrm{e}^{-\lambda t} W_{1}\left(\mu_{1}, \mu_{2}\right), \quad t \geq 0, \mu_{1}, \mu_{2} \in \mathscr{P}\left(\mathbb{R}^{d}\right)
$$


(2) Let $P_{t}$ have a unique invariant probability measure $\mu$ such that the log-Sobolev inequality

$$
\mu\left(f^{2} \log f^{2}\right) \leq C \mu\left(|\nabla f|^{2}\right), \quad f \in C_{b}^{1}\left(\mathbb{R}^{d}\right), \mu\left(f^{2}\right)=1
$$

holds for some constant $C>0$. If there exists a constant $K>0$ such that

$$
\|\sigma(x)-\sigma(y)\|_{H S}^{2}+\langle b(x)-b(y), x-y\rangle \leq K|x-y|^{2}, \quad x, y \in \mathbb{R}^{d},
$$

then (2.3) holds for some constants $c, \lambda>0$ and $M=\mathbb{R}^{d}$.

(3) Let $P_{t}$ be ultracontractive and let (2.21) hold for some constant $K>0$. Then there exist a constant $\lambda>0$ such that for any $p \in[1, \infty)$, condition $(2.18)$ implies

$$
W_{p}\left(\mu_{1} P_{t}, \mu_{2} P_{t}\right) \leq c \mathrm{e}^{-\lambda t} W_{p}\left(\mu_{1}, \mu_{2}\right), \quad t \geq 0, \mu_{1}, \mu_{2} \in \mathscr{P}\left(\mathbb{R}^{d}\right)
$$

for some constant $c=c(p)>0$.

According to [22, Lemma 3.3], we have

$$
\left\|\sigma_{0}(x)-\sigma_{0}(y)\right\|^{2} \leq \frac{1}{4 \lambda_{0}}\left\|\left(\sigma \sigma^{*}\right)(x)-\left(\sigma \sigma^{*}\right)(y)\right\|_{H S}^{2}, \quad x, y \in \mathbb{R}^{d} .
$$

Combining this with $\|\cdot\|_{H S}^{2} \leq d\|\cdot\|^{2}$, we see that (2.19) follows from the following more explicit condition:

$$
\begin{aligned}
& \frac{d-1}{4 \lambda_{0}}\left\|\left(\sigma \sigma^{*}\right)(x)-\left(\sigma \sigma^{*}\right)(y)\right\|_{H S}^{2}+\langle b(x)-b(y), x-y\rangle \\
& \leq\left\{\left(K_{1}+K_{2}\right) 1_{\left\{|x-y| \leq r_{0}\right\}}-K_{2}\right\}|x-y|^{2}, \quad x, y \in \mathbb{R}^{d} .
\end{aligned}
$$

\section{Preparations}

This section includes some propositions which will be used to prove the results introduced in Section 2. We first recall a link between the Wasserstein distance and gradient estimates due to [17], then deduce the hyperboundedness and the exponential convergence in entropy from the log-Sobolev inequality for non-symmetric diffusion semigroups, and finally prove the exponential contraction in gradient for ultracontractive semigroups in a general framework including both diffusion and jump Markov semigroups.

\subsection{Wasserstein distance and gradient inequalities}

Let $(E, \rho)$ be a geodesic Polish space, i.e. it is a Polish space and for any two different points $x, y \in E$, there exists a continuous curve $\gamma:[0,1] \rightarrow E$ such that $\gamma_{0}=x, \gamma_{1}=y$ and $\rho\left(\gamma_{s}, \gamma_{t}\right)=|s-t| \rho(x, y)$ for $s, t \in[0,1]$. Then for any $f \in \operatorname{Lip}_{b}(E)$, the class of bounded Lipschitz functions on $E$, the length of gradient

$$
|\nabla f|(x):=\limsup _{\rho(x, y) \downarrow 0} \frac{|f(x)-f(y)|}{\rho(x, y)}, \quad x \in E
$$


is measurable. Moreover, let $P(x, \mathrm{~d} y)$ be a Markov transition kernel and define the Markov operator

$$
P f(x):=\int_{E} f(y) P(x, \mathrm{~d} y), \quad x \in E, f \in \mathscr{B}_{b}(E) .
$$

For any $\Phi \in \overline{\mathscr{N}} \backslash\left\{\Phi_{\infty}\right\}$, consider the Young norm induced by $\Phi$ with respect to $P$

$$
\|f\|_{L_{*}^{\Phi}(P)}(x):=\sup \left\{P(f g)(x): g \in \mathscr{B}_{b}(E), P \Phi(|g|)(x) \leq 1\right\}, \quad x \in E, f \in \mathscr{B}_{b}(E),
$$

and set $\|f\|_{L_{*}^{\Phi_{\infty}(P)}}(x)=P|f|(x)$. Then $\|\cdot\|_{L_{*}^{\Phi_{p}}}=\|\cdot\|_{L^{\Phi_{q}}}$ for $p \in[1, \infty], q=\frac{p}{p-1}$. The following result follows from [17, Theorem 2.2, Remark 2 and Remark 3].

Proposition 3.1 ([17]). For any constant $C>0$ and $\Phi \in \overline{\mathscr{N}}$, the following statements are equivalent to each other:

(1) $|\nabla P f| \leq C\|\nabla f\|_{L_{*}^{\Phi}(P)}$ for $f \in \operatorname{Lip}_{b}(E)$.

(2) $W_{\Phi}\left(\delta_{x} P, \delta_{y} P\right) \leq C \rho(x, y), \quad x, y \in E$.

When $\Phi=\Phi_{p}$ for $p \in[1, \infty]$, they are also equivalent to

(3) $W_{p}\left(\mu_{1} P, \mu_{2} P\right) \leq C W_{p}\left(\mu_{1}, \mu_{2}\right), \quad \mu_{1}, \mu_{2} \in \mathscr{P}(E)$.

\subsection{Hyperboundedness and exponential convergence in entropy}

When $P_{t}$ is symmetric, it is well known that the hyperbounddeness, exponential convergence in entropy and the log-Sobolev inequality are equivalent each other, see [6, 36] and references within. In the non-symmetric case, the log-Sobolev inequality implies the former two properties if the generator $L$ and the symmetric part of the Dirichlet form $\mathscr{E}$ satisfy

$$
\begin{aligned}
& -\mu((1+\log f) L f) \geq c_{0} \mathscr{E}(\sqrt{f}, \sqrt{f}) \text { and } \\
& -\mu\left(f^{p-1} L f\right)=\frac{c_{0}(p-1)}{p^{2}} \mathscr{E}\left(f^{\frac{p}{2}}, f^{\frac{p}{2}}\right), \quad p>1, f \in \mathscr{D}
\end{aligned}
$$

for some constant $c_{0}>0$ and a reasonable class $\mathscr{D}$ of non-negative bounded functions, which is stable under $P_{t}$ and dense in $L_{+}^{p}(\mu):=\left\{f \in L^{p}(\mu): f \geq 0\right\}$ for any $p \geq 1$, see e.g. [14]. In applications, it may be not easy to figure out the class $\mathscr{D}$ such that (3.2) holds. But in general this condition can be replaced by the following approximation formula Lemma 3.2 in the spirit of $[26]$.

Now, consider the (Neumann) semigroup $P_{t}$ generated by $L:=\Delta+Z$ for a locally bounded vector field $Z$ such that $P_{t}$ has a unique invariant probability measure $\mu$. Let

$$
\mathscr{D}_{0}=\left\{f \in C_{0}^{\infty}(M): f \text { satisfies the Neumann condition if } \partial M \neq \emptyset\right\} .
$$

Then $\left(L, \mathscr{D}_{0}\right)$ is dissipative (thus, closable) in $L^{1}(\mu)$ with closure $\left(L, \mathscr{D}_{1}(L)\right)$ generating $P_{t}$ in $L^{1}(\mu)$, see e.g. [29] and references therein. Let

$$
\mathscr{D}=\left\{f \in \mathscr{D}_{1}(L) \cap L^{\infty}(\mu): f \geq 0\right\} .
$$


Lemma 3.2. Let $f \in \mathscr{D}$ and $\psi \in C_{b}^{\infty}\left(\left[\operatorname{ess}_{\mu} \inf f, \infty\right)\right)$. There exists a sequence $\left\{f_{n}\right\}_{n \geq 1} \subset \mathscr{D}_{0}$ with $\inf f_{n}=\operatorname{ess}_{\mu} \inf f$ such that $f_{n} \rightarrow f$ in $L^{m}(\mu)$ for any $m \geq 1, L f_{n} \rightarrow L f$ in $L^{1}(\mu)$, and

$$
\mu(\psi(f) L f)=-\lim _{n \rightarrow \infty} \mu\left(\psi^{\prime}\left(f_{n}\right)\left|\nabla f_{n}\right|^{2}\right) .
$$

Proof. Since $f \in \mathscr{D} \subset \mathscr{D}_{1}(L) \cap L^{\infty}(\mu)$, there exists a uniformly bounded sequence $\left\{f_{n}\right\}_{n \geq 1} \subset$ $\mathscr{D}_{0}$ such that inf $f_{n}=\operatorname{ess}_{\mu} \inf f$ and $f_{n} \rightarrow f, L f_{n} \rightarrow L f$ in $L^{1}(\mu)$. By the uniform boundedness, $f_{n} \rightarrow f$ in $L^{m}(\mu)$ for any $m \geq 1$. Since $\psi \in C_{b}^{\infty}\left(\left[\inf f_{n}, \infty\right)\right)$,

$$
g_{n}:=\int_{\inf f_{n}}^{f_{n}} \psi(s) \mathrm{d} s \in \mathscr{D}_{c}:=\left\{g+c: c \in \mathbb{R}, g \in \mathscr{D}_{0}\right\} \subset \mathscr{D}_{1}(L) .
$$

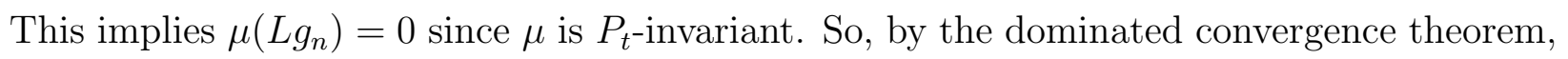

$$
\mu(\psi(f) L f)=\lim _{n \rightarrow \infty} \mu\left(\psi\left(f_{n}\right) L f_{n}\right)=\lim _{n \rightarrow \infty} \mu\left(L g_{n}-\psi^{\prime}\left(f_{n}\right)\left|\nabla f_{n}\right|^{2}\right)=-\lim _{n \rightarrow \infty} \mu\left(\psi^{\prime}\left(f_{n}\right)\left|\nabla f_{n}\right|^{2}\right) .
$$

Proposition 3.3. Let $Z$ be a locally bounded vector field such that the (Neumann) semigroup $P_{t}$ generated by $L:=\Delta+Z$ has a unique invariant probability measure $\mu$.

(1) If the super log-Sobolev inequality

$$
\mu\left(f^{2} \log f^{2}\right) \leq r \mu\left(|\nabla f|^{2}\right)+\beta(r), \quad r>0, \quad f \in C_{b}^{1}(M), \mu\left(f^{2}\right)=1 .
$$

holds for some $\beta \in C((0, \infty) ;(0, \infty))$, then for any constants $q>p \geq 1$ and $\gamma \in$ $C((p, q) ;(0, \infty))$ such that $t:=\int_{p}^{q} \frac{\gamma(r)}{r} \mathrm{~d} r<\infty$, there holds

$$
\left\|P_{t}\right\|_{L^{p}(\mu) \rightarrow L^{q}(\mu)} \leq \exp \left[\int_{p}^{q} \frac{\beta\left(4 \gamma(r)\left(1-r^{-1}\right)\right)}{r^{2}} \mathrm{~d} r\right] .
$$

(2) If the log-Sobolev inequality

$$
\mu\left(f^{2} \log f^{2}\right) \leq C \mu\left(|\nabla f|^{2}\right)+\mu\left(f^{2}\right) \log \mu\left(f^{2}\right), \quad f \in C_{b}^{1}(M)
$$

holds for some constant $C>0$, then

$$
\mu\left(\left(P_{t} g\right) \log P_{t} g\right) \leq \mathrm{e}^{-4 t / C} \mu(g \log g), \quad g \in \mathscr{B}_{b}(M), g \geq 0, \mu(g)=1 .
$$

Proof. (1) According to Lemma 3.2, for any $f \in \mathscr{D}$ and $p>1$, there exists $\left\{f_{n}\right\}_{n \geq 1} \subset \mathscr{D}_{0}$ such that $f_{n}^{\frac{2}{p}} \rightarrow f$ in $L^{m}(\mu)$ for all $m \geq 1$, and

$$
-\mu\left(f^{p-1} L f\right)=\frac{4(p-1)}{p^{2}} \limsup _{n \rightarrow \infty} \mu\left(\left|\nabla f_{n}\right|^{2}\right) .
$$


Applying (3.3) to $f_{n}$ and using (3.5), we obtain

$$
\begin{aligned}
& p \mu\left(f^{p} \log f\right)=\lim _{n \rightarrow \infty} \mu\left(f_{n}^{2} \log f_{n}^{2}\right) \leq r \liminf _{n \rightarrow \infty} \mu\left(\left|\nabla f_{n}\right|^{2}\right)+\beta(r) \\
& \leq \frac{r p^{2}}{4(p-1)}\left(-\mu\left(f^{p-1} L f\right)+\frac{4 \beta(r)(p-1)}{r p^{2}}\right), \quad r>0 .
\end{aligned}
$$

Set $c(p)=\frac{r p}{4(p-1)}$, we have

$$
\frac{4 \beta(r)(p-1)}{r p^{2}}=\frac{\beta\left(4 c(p)\left(1-p^{-1}\right)\right)}{p c(p)}, \quad p>1,
$$

so that the above inequality becomes

$$
\mu\left(f^{p} \log f\right) \leq c(p)\left(-\mu\left(f^{p-1} L f\right)+\gamma(p)\right), \quad p>1, f \in \mathscr{D}
$$

for $\gamma(p):=\frac{\beta\left(4 c(p)\left(1-p^{-1}\right)\right)}{p c(p)}$. Noting that $\mathscr{D}$ is $P_{t}$-invariant (i.e. $P_{t} \mathscr{D} \subset \mathscr{D}$ ) and dense in $L_{+}^{p}(\mu)$ for any $p \geq 1$, the desired assertion follows from the proof of [14, Corollary 3.13].

(2) It suffices to prove for $g \in \mathscr{D}$ with inf $g>0$. Applying Lemma 3.2 to $f=P_{t} g$ and $\psi(s)=1+\log s$, and using (3.4), we obtain

$$
\begin{aligned}
& \frac{\mathrm{d}}{\mathrm{d} t} \mu\left(\left(P_{t} g\right) \log P_{t} g\right)=\mu\left(\left(1+\log P_{t} g\right) L P_{t} g\right)=-4 \lim _{n \rightarrow \infty} \mu\left(\left|\nabla \sqrt{f_{n}}\right|^{2}\right) \\
& \leq-\frac{4}{C} \liminf _{n \rightarrow \infty} \mu\left(f_{n} \log f_{n}\right)=-\frac{4}{C} \mu\left(\left(P_{t} g\right) \log P_{t} g\right), \quad t \geq 0
\end{aligned}
$$

This implies the desired exponential estimate.

\subsection{Exponential contraction in gradient}

In this part, we consider a general framework including both diffusion and jump processes. Let $(E, \mathscr{F}, \mu)$ be a separable complete probability space, and let $P_{t}$ be a Markov semigroup on $L^{2}(\mu)$ with $\mu$ as invariant probability measure. Let $(L, \mathscr{D}(L))$ be the generator of $P_{t}$ in $L^{2}(\mu)$. We assume that there exists an algebra $\mathscr{A} \subset \mathscr{D}(L)$ such that

(i) $1 \in \mathscr{A}, \mathscr{A}$ is dense in $L^{2}(\mu)$ and the algebra induced by

$$
\mathscr{D}:=\left\{P_{s} f: s \geq 0, f \in \mathscr{A}\right\}
$$

is contained in $\mathscr{D}(L)$.

(ii) $\Gamma(f, g):=\frac{1}{2}(L(f g)-f L g-g L f)$ gives rise to a non-degenerate positive definite bilinear form on $\mathscr{D} \times \mathscr{D}$; i.e., for any $f \in \mathscr{D}, \Gamma(f, f) \geq 0$ and it equals to 0 if and only if $f$ is constant. 
In particular, when $P_{t}$ is the (Neumann) semigroup generated by $L:=\Delta+Z$ on $M$ with $\mathrm{Ric}_{Z}$ bounded below, the assumption holds for

$$
\mathscr{A}:=\left\{f+c: f \in C_{0}^{\infty}(M) \text { satisfying the Neumann condition if } \partial M \neq \emptyset, c \in \mathbb{R}\right\} .
$$

Under the above conditions,

$$
\mathscr{E}(f, g):=\mu(\Gamma(f, g)), \quad f, g \in \mathscr{A}
$$

is closable and the closure $(\mathscr{E}, \mathscr{D}(\mathscr{E}))$ is a conservative symmetric Dirichlet form. Although $P_{t}$ is not associated to $(\mathscr{E}, \mathscr{D}(\mathscr{E}))$ when it is non-symmetric, we have

$$
\frac{\mathrm{d}}{\mathrm{d} t} \mu\left(\left(P_{t} f\right)^{2}\right)=-2 \mathscr{E}\left(P_{t} f, P_{t} f\right), \quad t \geq 0, f \in \mathscr{D} .
$$

If $\left\|P_{t}\right\|_{L^{1}(\mu) \rightarrow L^{\infty}(\mu)}<\infty$, then $P_{t}$ has a heat kernel $p_{t}(x, y)$ with respect to $\mu$, i.e.

$$
P_{t} f=\int_{E} p_{t}(\cdot, y) f(y) \mu(\mathrm{d} y), \quad f \in L^{2}(\mu),
$$

and

$$
\operatorname{ess}_{\mu \times \mu} \sup p_{t}=\left\|P_{t}\right\|_{L^{1}(\mu) \rightarrow L^{\infty}(\mu)}<\infty .
$$

We consider the "gradient" length $\left|\nabla_{\Gamma} f\right|=\sqrt{\Gamma(f, f)}$ induced by $\Gamma$. Note that for jump processes the length is non-local and thus essentially different from the usual gradient length. As shown below that estimates of $\left|\nabla_{\Gamma} P_{t}\right|$ have a close link to functional inequalities of the associated Dirichlet form.

Proposition 3.4. Assume that there exist $t_{1}>0$ and $\eta \in C([0, \infty) ;(0, \infty))$ such that

$$
\left\|P_{t_{1}}\right\|_{L^{1}(\mu) \rightarrow L^{\infty}(\mu)}<\infty, \quad\left|\nabla_{\Gamma} P_{t} f\right|^{2} \leq \eta(t) P_{t}\left|\nabla_{\Gamma} f\right|^{2}, t \geq 0, f \in \mathscr{D} .
$$

Then there exist constants $c, \lambda, t_{2}>0$ such that for any $q \geq 1$ and $\eta_{q} \in C([0, \infty) ;(0, \infty))$, the gradient estimate

$$
\left|\nabla_{\Gamma} P_{t} f\right|^{2} \leq \eta_{q}(t)\left(P_{t}\left|\nabla_{\Gamma} f\right|^{q}\right)^{\frac{2}{q}}, t \geq 0, f \in \mathscr{D}
$$

implies

$$
\left\|\nabla_{\Gamma} P_{t} f\right\|_{L^{\infty}(\mu)}^{2} \leq\left(c \sup _{\left[0, t_{2}\right]} \eta_{q}\right) \mathrm{e}^{-\lambda t} \operatorname{ess}_{\mu} \inf \left(P_{t}\left|\nabla_{\Gamma} f\right|^{q}\right)^{\frac{2}{q}}, \quad t \geq t_{2}, f \in \mathscr{D} .
$$

Proof. (a) We first prove

$$
\mathscr{E}\left(P_{t} f, P_{t} f\right) \leq C \mathrm{e}^{-\lambda t} \mathscr{E}(f, f), \quad f \in \mathscr{D}, t \geq 0
$$

for some constants $C, \lambda>0$. By the second inequality in (3.7), for any $t>0$ and $f \in \mathscr{D}$ we have

$$
\frac{\mathrm{d}}{\mathrm{d} s} P_{s}\left(P_{t-s} f\right)^{2}=2 P_{s}\left|\nabla_{\Gamma} P_{t-s} f\right|^{2} \leq 2 \eta(t-s) P_{t}\left|\nabla_{\Gamma} f\right|^{2}, \quad s \in[0, t] .
$$


Integrating both sides over $[0, t]$ leads to

$$
P_{t} f^{2} \leq\left(P_{t} f\right)^{2}+C(t) P_{t}\left|\nabla_{\Gamma} f\right|^{2}, \quad C(t):=2 \int_{0}^{t} \eta(s) \mathrm{d} s, t>0 .
$$

Taking $t=t_{1}$ and noting that $\mu$ is the invariant probability measure of $P_{t}$, we obtain

$$
\mu\left(f^{2}\right) \leq C\left(t_{1}\right) \mathscr{E}(f, f)+\left\|P_{t_{1}}\right\|_{1 \rightarrow \infty}^{2} \mu(|f|)^{2}, \quad f \in \mathscr{D} .
$$

Since $\mathscr{D}(\mathscr{E})$ is the closure of $\mathscr{D}$ under the $\mathscr{E}_{1}$-norm, this inequality also holds for $f \in \mathscr{D}(\mathscr{E})$. By condition (ii), the symmetric Dirichlet form is irreducible. So, according to [41, Corollary 1.2] the defective Poincaré inequality (3.11) implies the Poincaré inequality

$$
\mu\left(f^{2}\right) \leq \frac{1}{\lambda} \mathscr{E}(f, f)+\mu(f)^{2}, \quad f \in \mathscr{D}(\mathscr{E})
$$

for some constant $\lambda>0$. By (3.6) and that $\mathscr{D}$ is dense in $L^{2}(\mu)$, the Poincaré inequality is equivalent to

$$
\left\|P_{t} f-\mu(f)\right\|_{2} \leq \mathrm{e}^{-\lambda t}\|f-\mu(f)\|_{2}, \quad t \geq 0, f \in L^{2}(\mu) .
$$

On the other hand, by the second inequality in (3.7), for any $t>0$ and $f \in \mathscr{D}$ we have

$$
\frac{\mathrm{d}}{\mathrm{d} s} P_{s}\left(P_{t-s} f\right)^{2}=2 P_{s}\left|\nabla_{\Gamma} P_{t-s} f\right|^{2} \geq \frac{2}{\eta(s)}\left|\nabla_{\Gamma} P_{t} f\right|^{2}, \quad s \in[0, t] .
$$

So,

$$
\left|\nabla_{\Gamma} P_{t} f\right|^{2} \leq \frac{P_{t} f^{2}-\left(P_{t} f\right)^{2}}{2 \int_{0}^{t} \eta(s)^{-1} \mathrm{~d} s}, \quad t>0, f \in \mathscr{D} .
$$

Using $P_{t} f-\mu(f)$ to replace $f$ and integrating with respect to $\mu$, we obtain

$$
\mathscr{E}\left(P_{2 t} f, P_{2 t} f\right) \leq \frac{\left\|P_{t} f-\mu(f)\right\|_{2}^{2}}{2 \int_{0}^{t} \eta(s)^{-1} \mathrm{~d} s}, \quad t>0, f \in \mathscr{D} .
$$

Combining this with (3.13) and (3.12) we arrive at

$$
\mathscr{E}\left(P_{t} f, P_{t} f\right) \leq c_{1} \mathrm{e}^{-\lambda t} \mathscr{E}(f, f), \quad t \geq 1, f \in \mathscr{D}
$$

for some constant $c_{1}>0$; that is, (3.10) holds for $t>1$. Finally, (3.7) implies (3.10) for $t \in[0,1]$.

(b) Next, we intend to find out a constant $t_{0} \geq t_{1}$ such that

$$
\frac{1}{2} \leq p_{t} \leq 2, \quad(\mu \times \mu) \text {-a.e. }, t \geq t_{0} .
$$

Indeed, by (3.13) and the first inequality in (3.7), we obtain

$$
\left|\int_{E}\left(p_{t+2 t_{1}}(\cdot, y)-1\right) f(y) \mu(\mathrm{d} y)\right|=\left|P_{t_{1}}\left(P_{t+t_{1}} f-\mu(f)\right)\right|
$$




$$
\leq c_{0} \mu\left(\left|P_{t+t_{1}} f-\mu(f)\right|\right) \leq c_{0} \mathrm{e}^{-\lambda t}\left\|P_{t_{1}} f-\mu(f)\right\|_{2} \leq c_{0}^{2} \mathrm{e}^{-\lambda t} \mu(|f|), \quad \mu \text {-a.e., } t \geq 0,
$$

where $c_{0}:=\left\|P_{t_{1}}\right\|_{L^{1}(\mu) \rightarrow L^{\infty}(\mu)}$. This implies the desired assertion for $t_{0}>0$ such that $c_{0}^{2} \mathrm{e}^{-\lambda t_{0}} \leq$ $\frac{1}{2}$.

(c) Finally, combining (3.7), (3.14), (3.10) and (3.8), we obtain

$$
\begin{aligned}
& \left\|\nabla_{\Gamma} P_{t+2 t_{0}} f\right\|_{L^{\infty}(\mu)}^{2} \leq c_{1}\left\|P_{t_{0}}\left|\nabla_{\Gamma} P_{t+t_{0}} f\right|^{2}\right\|_{L^{\infty}(\mu)} \leq 2 c_{1} \mathscr{E}\left(P_{t+t_{0}} f, P_{t+t_{0}} f\right) \\
& \leq c_{2} \mathrm{e}^{-\lambda t} \mathscr{E}\left(P_{t_{0}} f, P_{t_{0}} f\right) \leq c_{2} \eta_{q}\left(t_{0}\right) \mathrm{e}^{-\lambda t} \mu\left(\left(P_{t_{0}}\left|\nabla_{\Gamma} f\right|^{q}\right)^{\frac{2}{q}}\right) \\
& \leq c_{3} \eta_{q}\left(t_{0}\right) \mathrm{e}^{-\lambda t} \operatorname{ess}_{\mu} \inf \left(P_{t+2 t_{0}}\left|\nabla_{\Gamma} f\right|^{q}\right)^{\frac{2}{q}}
\end{aligned}
$$

for some constants $c_{1}, c_{2}, c_{3}>0$. Then (3.9) holds for $t_{2}=2 t_{0}$.

\section{Proofs of Theorem 2.1 and Corollary 2.2}

The first assertion is a generalization of the main result in [18] where $M=\mathbb{R}^{d}$ is considered. As in [18], the key point of the proof is to construct a coupling by parallel transform for long distance but by reflection for short distance. The only difference is that we are working on a non-flat Riemannian manifold for which the curvature term appears in calculations. Since Itô's formula of the distance process has been well developed for couplings by both parallel displacement and reflection, the proof is also straightforward.

The proofs of the other two assertions are based on the log-Sobolev inequality and the log-Harnack inequality derived in [25] and [39] respectively for bounded below $\mathrm{Ric}_{Z}$.

Proof of Theorem 2.1. (a) For two different points $x, y \in M$, let $P_{x, y}: T_{x} M \rightarrow T_{y} M$ be the parallel displacement along the minimal geodesic $\gamma:[0, \rho(x, y)] \rightarrow M$ from $x$ to $y$, and let $M_{x, y}:=P_{x, y}-2\left\langle\cdot, \dot{\gamma}_{0}\right\rangle \dot{\gamma}_{\rho(x, y)}: T_{x} M \rightarrow T_{y} M$ be the mirror reflection. Both maps are smooth in $(x, y)$ outside the cut-locus Cut $(M)$. According to [15] and [32], the appearance of the cut-locus and/or a convex boundary helps for the success of coupling, i.e. it makes the distance between two marginal processes smaller. So, for simplicity, we may and do assume that both the cut-locus and the boundary are empty, see [3, Section 3] or [36, Chapter 2] for details.

Now, let $X_{t}$ solve the SDE

$$
\mathrm{d}_{I} X_{t}=\sqrt{2} u_{t} \mathrm{~d} B_{t}+Z\left(X_{t}\right) \mathrm{d} t, \quad X_{0}=x,
$$

where $\mathrm{d}_{I}$ denotes the Itô differential introduced in [13] on Riemannian manifolds, $B_{t}$ is the $d$-dimensional Brownian motion, and $u_{t}$ is the horizontal lift of $X_{t}$ to the frame bundle $O(M)$. Then $X_{t}$ is a diffusion process generated by $L$. To construct the coupling by reflection for short distance and parallel displacement for long distance, we introduce a cut-off function $h \in C^{1}([0, \infty))$ which is decreasing such that $h(r)=1$ for $r \leq r_{0}, h(r)=0$ for $r \geq$ $r_{0}+1$, and $\sqrt{1-h^{2}}$ is also in $C^{1}$, see e.g. [43, (3.1)] for a concrete example. To construct the coupling in the above spirit, we split the noise into two parts, i.e. to replace $\mathrm{d} B_{t}$ by $h\left(\rho\left(X_{t}, Y_{t}\right)\right) \mathrm{d} B_{t}^{\prime}+\sqrt{1-h\left(\rho\left(X_{t}, Y_{t}\right)\right)^{2}} \mathrm{~d} B_{t}^{\prime \prime}$ for two independent Brownian motions $B_{t}^{\prime}$ and 
$B_{t}^{\prime \prime}$, then make reflection for the $B_{t}^{\prime}$ part and parallel displacement for the $B_{t}^{\prime \prime}$ part. More precisely, let $\left(X_{t}, Y_{t}\right)$ solve the following $\mathrm{SDE}$ on $M \times M$ for $\left(X_{0}, Y_{0}\right)=(x, y)$ :

$$
\begin{aligned}
& \mathrm{d}_{I} X_{t}=\sqrt{2}\left(h\left(\rho\left(X_{t}, Y_{t}\right)\right) u_{t} \mathrm{~d} B_{t}^{\prime}+\sqrt{1-h\left(\rho\left(X_{t}, Y_{t}\right)\right)^{2}} u_{t} \mathrm{~d} B_{t}^{\prime \prime}\right)+Z\left(X_{t}\right) \mathrm{d} t \\
& \mathrm{~d}_{I} Y_{t}=\sqrt{2}\left(h\left(\rho\left(X_{t}, Y_{t}\right)\right) M_{X_{t}, Y_{t}} u_{t} \mathrm{~d} B_{t}^{\prime}+\sqrt{1-h\left(\rho\left(X_{t}, Y_{t}\right)\right)^{2}} P_{X_{t}, Y_{t}} u_{t} \mathrm{~d} B_{t}^{\prime \prime}\right)+Z\left(Y_{t}\right) \mathrm{d} t
\end{aligned}
$$

Since the coefficients of the SDE are at least $C^{1}$ outside the diagonal $\{(z, z): z \in M\}$, it has a unique solution up to the coupling time

$$
T:=\inf \left\{t \geq 0: X_{t}=Y_{t}\right\}
$$

We then let $X_{t}=Y_{t}$ for $t \geq T$ as usual. By the second variational formula and the index lemma (see e.g. the proof of $[37$, Lemma 2.3] and $[32,(2.4)])$, the process $\rho_{t}:=\rho\left(X_{t}, Y_{t}\right)$ satisfies

$$
\mathrm{d} \rho_{t} \leq 2 \sqrt{2} h\left(\rho_{t}\right) \mathrm{d} b_{t}+I_{Z}\left(X_{t}, Y_{t}\right) \mathrm{d} t, \quad t \leq T
$$

for some one-dimensional Brownian motion $b_{t}$. Thus, by condition (1.8),

$$
\mathrm{d} \rho_{t} \leq 2 \sqrt{2} h\left(\rho_{t}\right) \mathrm{d} b_{t}+\left\{\left(K_{1}+K_{2}\right) 1_{\left\{\rho_{t} \leq r_{0}\right\}}-K_{2}\right\} \rho_{t} \mathrm{~d} t, \quad t \leq T .
$$

Since $h\left(\rho_{t}\right)=0$ for $\rho_{t} \geq r_{0}+1$ while $\mathrm{d} \rho_{t}<0$ when $\rho_{t} \geq r_{0}+1$, this implies

$$
\rho_{t} \leq\left(r_{0}+1\right) \vee \rho_{0} \leq 1+r_{0}+\rho(x, y) .
$$

On the other hand, since $h\left(\rho_{t}\right)=1$ for $\rho_{t} \leq r_{0}$, as observed in [18] we have

$$
\mathbb{E} \rho_{t} \leq c \mathrm{e}^{-\lambda t} \rho(x, y), \quad t \geq 0
$$

for some constants $c, \lambda>0$. Indeed, let

$$
\bar{\rho}_{t}=\varepsilon \rho_{t}+1-\mathrm{e}^{-N \rho_{t}}, \quad N=\frac{r_{0}}{2}\left(K_{1}+K_{2}\right), \varepsilon=N \mathrm{e}^{-N r_{0}} .
$$

Then

$$
\varepsilon \rho_{t} \leq \bar{\rho}_{t} \leq(N+\varepsilon) \rho_{t}, \quad \frac{4 N^{2}}{r\left(\varepsilon \mathrm{e}^{N r}+N\right)} \geq K_{1}+K_{2} \text { for } r \in\left(0, r_{0}\right]
$$

so that (4.1) and Itô's formula lead to

$$
\begin{aligned}
\mathrm{d} \bar{\rho}_{t} \leq & 2 \sqrt{2}\left(\varepsilon+N \mathrm{e}^{-N \rho_{t}}\right) h\left(\rho_{t}\right) \mathrm{d} b_{t} \\
& +\left(\varepsilon+N \mathrm{e}^{-N \rho_{t}}\right)\left\{\left(K_{1}+K_{2}\right) 1_{\left\{\rho_{t} \leq r_{0}\right\}}-K_{2}-\frac{4 N^{2}}{\rho_{t}\left(\varepsilon \mathrm{e}^{N \rho_{t}}+N\right)} 1_{\left\{\rho_{t} \leq r_{0}\right\}}\right\} \rho_{t} \mathrm{~d} t \\
\leq & 2 \sqrt{2}\left(\varepsilon+N \mathrm{e}^{-N \rho_{t}}\right) h\left(\rho_{t}\right) \mathrm{d} b_{t}-c_{1} \bar{\rho}_{t} \mathrm{~d} t, \quad t \leq T
\end{aligned}
$$

for some constant $c_{1}$. This implies $\mathbb{E} \bar{\rho}_{t} \leq \bar{\rho}_{0} \mathrm{e}^{-c_{1} t}$. Then (4.3) holds for some constants $c, \lambda>0$. Combining (4.2) with (4.3) we arrive at

$$
\mathbb{E} \Phi\left(\rho_{t} / r\right) \leq \sup _{s \in\left(0,1+r_{0}+\rho_{0}\right]} \frac{\Phi(s / r)}{s} \mathbb{E} \rho_{t} \leq c \mathrm{e}^{-\lambda t} \rho(x, y) \sup _{s \in\left(0,1+r_{0}+\rho_{0}\right]} \frac{\Phi(s / r)}{s}
$$


So,

$$
\begin{aligned}
& W_{\Phi}\left(\delta_{x} P_{t}, \delta_{y} P_{t}\right) \leq\left\|\rho_{t}\right\|_{L^{\Phi}(\mathbb{P})}=\inf \left\{r>0: \mathbb{E} \Phi\left(\rho_{t} / r\right) \leq 1\right\} \\
& \leq \inf \left\{r>0: \sup _{s \in\left(0,1+r_{0}+\rho(x, y)\right]} \frac{\Phi\left(\frac{s}{r}\right)}{s} \leq \frac{\mathrm{e}^{\lambda t}}{c \rho(x, y)}\right\},
\end{aligned}
$$

which proves (2.1). Therefore, the proof of (1) is finished since the second inequality therein is a simple consequence of $(2.1)$.

(b) To prove the log-Sobolev inequality (3.4), we follow the line of [37] to establish the dimension-free Harnack inequality using coupling by change of measures. Let $\left(x_{t}, y_{t}\right)$ be the coupling constructed in [37] before Lemma 2.3 therein with $Z$ replacing $\nabla V$ and

$$
\xi_{t}=C+\frac{\rho(x, y)}{T}
$$

for a constant $C>0$. We first prove that when $C$ is large enough our condition implies $x_{T}=y_{T}$ as in [37, Lemma 2.3]. Indeed, by (1.8) and [37, (2.4)], we have

$$
\mathrm{d} \rho\left(x_{t}, y_{t}\right)=\left\{I_{Z}\left(x_{t}, y_{t}\right)-\xi_{t}\right\} \mathrm{d} t \leq\left\{\left(K_{1}+K_{2}\right) r_{0}-C-\frac{\rho(x, y)}{T}\right\} \mathrm{d} t, \quad t<\tau,
$$

where $\tau:=\inf \left\{t \geq 0: x_{t}=y_{t}\right\}$ is the coupling time. Taking $C=\left(K_{1}+K_{2}\right) r_{0}$ we obtain

$$
\mathrm{d} \rho\left(x_{t}, y_{t}\right) \leq-\frac{\rho(x, y)}{T} \mathrm{~d} t, \quad t<\tau,
$$

so that $\tau \leq T$ as desired.

Next, let $R$ be given in the proof of [37, Proposition 3.1] for the present $\xi_{t}$ in (4.4):

$$
R=\exp \left[-\frac{1}{\sqrt{2}} \int_{0}^{\tau} \mathrm{d} M_{t}-\frac{1}{4} \int_{0}^{\tau} \xi_{t}^{2} \mathrm{~d} t\right],
$$

where $M_{t}$ is a martingale with $\mathrm{d}\langle M\rangle_{t}=\frac{1}{2} \xi_{t}^{2} \mathrm{~d} t$. Then there exists a constant $C>0$ such that for any $\alpha>1$,

$$
\begin{aligned}
\mathbb{E} R^{\frac{\alpha}{\alpha-1}} & =\mathbb{E} \exp \left[-\frac{\alpha}{(\alpha-1) \sqrt{2}} \int_{0}^{\tau} \mathrm{d} M_{t}-\frac{\alpha^{2}}{4(\alpha-1)^{2}} \int_{0}^{\tau} \xi_{t}^{2} \mathrm{~d} t+\frac{\alpha}{4(\alpha-1)^{2}} \int_{0}^{\tau} \xi_{t}^{2} \mathrm{~d} t\right] \\
& \leq \exp \left[\frac{C \alpha}{(\alpha-1)^{2}}\left(T+\frac{\rho(x, y)^{2}}{T}\right)\right] .
\end{aligned}
$$

By $[37,(3.2)]$, this implies the Harnack inequality

$$
\left(P_{t} f(y)\right)^{\alpha} \leq P_{T} f^{\alpha}(x) \exp \left[\frac{C \alpha}{\alpha-1}\left(T+\frac{\rho(x, y)^{2}}{T}\right)\right], \quad 0 \leq f \in \mathscr{B}(M), x, y \in M, T>0 .
$$

Combining this with $\mu\left(\mathrm{e}^{\varepsilon \rho_{o}^{2}}\right)<\infty$, it is easy to see that $\left\|P_{T}\right\|_{2 \rightarrow 4}<\infty$ for large $T>0$. Since (1.8) implies $\operatorname{Ric}_{Z} \geq-\left(K_{1}+K_{2}\right)$, by the hyperboundedness and [25, Theorem 2.1], we have the defective log-Sobolev inequality

$$
\mu\left(f^{2} \log f^{2}\right) \leq C_{1} \mu\left(|\nabla f|^{2}\right)+C_{2}, \quad f \in C_{b}^{1}(M), \mu\left(f^{2}\right)=1
$$


for some constants $C_{1}, C_{2}>0$. Since the symmetric Dirichlet form $\mathscr{E}(f, g):=\mu(\langle\nabla f, \nabla g\rangle)$ with domain $H^{1,2}(\mu)$ is irreducible, according to [41] (see also [19]), the log-Sobolev inequality (3.4) holds for some constant $C>0$.

(c) When $\partial M=\emptyset$, the log-Sobolev inequality implies the Talagrand inequality

$$
W_{2}(f \mu, \mu)^{2} \leq \frac{C}{2} \mu(f \log f), \quad f \geq 0, \mu(f)=1,
$$

see $[5,35,21]$. Next, let $P_{t}^{*}$ be the adjoint of $P_{t}$ in $L^{2}(\mu)$. Since $\mu$ is $P_{t}$-invariant, $P_{t}^{*}$ is generated by $L^{*}:=\Delta+Z^{*}$ for the $C^{1}$-vector field $Z^{*}=2 \nabla V-Z$, where $V=\log \frac{\mathrm{d} \mu}{\mathrm{d} x}$. So, by Proposition 3.3 for $P_{t}^{*}$ in place of $P_{t}$, the $\log$-Sobolev inequality implies

$$
\mu\left(\left(P_{t}^{*} f\right) \log P_{t}^{*} f\right) \leq \mathrm{e}^{-4 t / C} \mu(f \log f), \quad t \geq 0, f \geq 0, \mu(f)=1 .
$$

Moreover, according to [39, Theorem 1.1], the curvature condition $\operatorname{Ric}_{Z} \geq-\left(K_{1}+K_{2}\right)=:-K$ is equivalent to the log-Harnack inequality

$$
P_{t}(\log f)(x) \leq \log P_{t} f(y)+\frac{K \rho(x, y)^{2}}{2\left(1-\mathrm{e}^{-2 K t}\right)}, \quad t \geq 0, x, y \in M, 0 \leq f \in \mathscr{B}_{b}(M) .
$$

By [42, Proposition 1.4.4(3)], this implies

$$
\mu\left(\left(P_{t}^{*} f\right) \log P_{t}^{*} f\right) \leq \frac{K}{2\left(1-\mathrm{e}^{-2 K t}\right)} W_{2}(f \mu, \mu)^{2}, \quad f \geq 0, \mu(f)=1, t>0 .
$$

Combining (4.5), (4.6) and (4.7), we obtain

$$
\begin{aligned}
& W_{2}\left((f \mu) P_{1+t}, \mu\right)^{2}=W_{2}\left(\left(P_{1+t}^{*} f\right) \mu, \mu\right)^{2} \leq \frac{C}{2} \mu\left(\left(P_{1+t}^{*} f\right) \log P_{1+t}^{*} f\right) \\
& \leq \frac{C}{2} \mathrm{e}^{-4 t / C} \mu\left(\left(P_{1}^{*} f\right) \log P_{1}^{*} f\right) \leq c_{1} \mathrm{e}^{-4 t / C} W_{2}(f \mu, \mu)^{2}, \quad t \geq 0, f \geq 0, \mu(f)=1
\end{aligned}
$$

for some constant $c_{1}>0$. Noting that $\operatorname{Ric}_{Z} \geq-K$ implies $\left|\nabla P_{t} f\right| \leq \mathrm{e}^{K t} P_{t}|\nabla f|$ (see e.g. [39]), by Proposition 3.1 we have

$$
W_{2}\left((f \mu) P_{t}, \mu\right)=W_{2}\left((f \mu) P_{t}, \mu P_{t}\right) \leq c_{2} W_{2}(f \mu, \mu), \quad t \in[0,1], f \geq 0, \mu(f)=1 .
$$

Combining with (4.8) yields

$$
W_{2}\left((f \mu) P_{t}, \mu\right) \leq c \mathrm{e}^{-\lambda t} W_{2}(f \mu, \mu), \quad t \geq 0, f \geq 0, \mu(f)=1
$$

for some constants $c, \lambda>0$. Therefore, the proof of (3) is finished.

Proof of Corollary 2.2. By Theorem 2.1, it suffices to verify condition (1.8) and to prove that $P_{t}$ has a unique invariant probability measure $\mu$ with $\mu\left(\mathrm{e}^{\varepsilon \rho_{o}^{2}}\right)<\infty$ for some constant $\varepsilon>0$.

Since Ric $\geq-\delta_{1},[37,(2.5)]$ applies to $K(x, y)=\delta_{1}$. So,

$$
I(x, y) \leq 2 \sqrt{\delta_{1}(d-1)} .
$$


On the other hand, by $\nabla Z \leq-\delta_{2}$ outside a compact set, there exist constants $c, r>0$ such that

$$
\nabla Z \leq-\delta_{2}+c 1_{\left\{\rho_{o} \leq r\right\}}
$$

Then, letting $\gamma:[0, \rho(x, y)] \rightarrow M$ be the minimal geodesic from $x$ to $y$ with $|\dot{\gamma}|=1$, we obtain

$$
\begin{aligned}
& \langle Z, \nabla \rho(\cdot, y)\rangle(x)+\langle Z, \nabla \rho(x, \cdot)\rangle(y)=\int_{0}^{\rho(x, y)} \frac{\mathrm{d}}{\mathrm{d} s}\left\langle Z\left(\gamma_{s}\right), \dot{\gamma}_{s}\right\rangle \mathrm{d} s \\
& =\int_{0}^{\rho(x, y)}\left\langle\nabla_{\dot{\gamma}_{s}} Z\left(\gamma_{s}\right), \dot{\gamma}_{s}\right\rangle \mathrm{d} s \leq-\delta_{2} \rho(x, y)+2 c r .
\end{aligned}
$$

Combining this with (4.9) we prove (1.8) for some constants $K_{1}, K_{2}>0$.

Next, by Ric $\geq-\delta_{1}$ and $\nabla Z \leq-\delta_{2}$ outside a compact set, there exist constants $c_{1}, c_{2}>0$ such that

$$
L \rho_{o}^{2} \leq c_{1}-c_{2} \rho_{0}^{2}
$$

for some constants $c_{1}, c_{2}>0$. So, when $\varepsilon>0$ is small enough,

$$
L \mathrm{e}^{\varepsilon \rho_{o}^{2}} \leq c(\varepsilon)-\gamma(\varepsilon) \mathrm{e}^{\varepsilon \rho_{o}^{2}}
$$

holds for some constants $c(\varepsilon), \delta(\varepsilon)>0$. By a standard argument this implies that $P_{t}$ has an invariant probability measure $\mu$ with $\mu\left(\mathrm{e}^{\varepsilon \rho_{o}^{2}}\right)<\infty$. The uniqueness of $\mu$ follows from the irreducibility and strong Feller property which is well known for the present framework.

\section{Proof of Theorem 2.3 and Corollary 2.4}

Proof of Theorem 2.3. (1) Since $\operatorname{Ric}_{Z} \geq-K$ for some constant $K \geq 0$, we have (see e.g. [39])

$$
\left|\nabla P_{t} f\right| \leq \mathrm{e}^{K t} P_{t}|\nabla f|, \quad f \in C_{b}^{1}(M) .
$$

Combining this with Proposition 3.4 for $q=1$ and noting that $P_{t}|\nabla f|$ is continuous, we obtain

$$
\left|\nabla P_{t} f\right| \leq c_{0} \mathrm{e}^{-\lambda t} P_{t}|\nabla f|, \quad t \geq t_{0}, f \in C_{b}^{1}(M)
$$

for some constants $c_{0}, \lambda, t_{0}>0$. Obviously, (3.1) implies

$$
\|\cdot\|_{L^{1}\left(P_{t}\right)} \leq \frac{\|\cdot\|_{L_{*}^{\Phi}\left(P_{t}\right)}}{\Phi^{-1}(1)}, \quad \Phi \in \overline{\mathscr{N}} .
$$

Then

$$
\left|\nabla P_{t} f\right| \leq \frac{c_{0}}{\Phi^{-1}(1)} \mathrm{e}^{-\lambda t}\|\nabla f\|_{L_{*}^{\Phi}\left(P_{t}\right)}, \quad t \geq 0, \Phi \in \overline{\mathscr{N}}, f \in C_{b}^{1}(M) .
$$

According to Proposition 3.1, this is equivalent to

$$
W_{\Phi}\left(\delta_{x} P_{t}, \delta_{y} P_{t}\right) \leq \frac{c_{0}}{\Phi^{-1}(1)} \mathrm{e}^{-\lambda t} \rho(x, y), \quad t \geq 0, x, y \in M .
$$


On the other hand, noting that

$$
\mathscr{C}\left(\delta_{x} P_{t}, \delta_{y} P_{t}\right) \ni \pi_{t}:=\left(\delta_{x} P_{t}\right) \times\left(\delta_{y} P_{t}\right) \leq\left\|P_{t}\right\|_{L^{1}(\mu) \rightarrow L^{\infty}(\mu)}^{2}(\mu \times \mu),
$$

we obtain

$$
W_{\Phi}\left(\delta_{x} P_{t}, \delta_{y} P_{t}\right) \leq\|\rho\|_{L^{\Phi}\left(\pi_{t}\right)} \leq G_{\Phi}(2 t), \quad t>0 .
$$

Combining this with (5.1) and the semigroup property, we arrive at

$$
W_{\Phi}\left(\delta_{x} P_{t}, \delta_{y} P_{t}\right) \leq \frac{c_{0}}{\Phi^{-1}(1)} \mathrm{e}^{-\lambda t / 2} W_{\Phi}\left(\delta_{x} P_{t / 2}, \delta_{y} P_{t / 2}\right) \leq \frac{c_{0}}{\Phi^{-1}(1)} \mathrm{e}^{-\lambda t / 2} G_{\Phi}(t) .
$$

This together with (5.1) implies (2.6) for some constants $c, \lambda>0$. Moreover, (2.8) follows from (2.6) according to Proposition 3.1.

(2) By Proposition 3.1, (2.9) implies

$$
\left|\nabla P_{t} f\right| \leq c \mathrm{e}^{-\lambda t} P_{t}|\nabla f|, \quad t \geq 0, f \in C_{b}^{1}(M)
$$

Then using the standard semigroup calculation of Bakry-Emery, this implies

$$
\begin{aligned}
& P_{t}\left(f^{2} \log f^{2}\right)-\left(P_{t} f^{2}\right) \log P_{t} f^{2}=\int_{0}^{t} \frac{\mathrm{d}}{\mathrm{d} s} P_{s}\left\{\left(P_{t-s} f^{2}\right) \log P_{t-s} f^{2}\right\} \mathrm{d} s \\
& =\int_{0}^{t} P_{s}\left(\frac{\left|\nabla P_{t-s} f^{2}\right|^{2}}{P_{t-s} f^{2}}\right) \mathrm{d} s \leq 4 c^{2} \int_{0}^{t} \mathrm{e}^{-2 \lambda(t-s)} P_{s}\left(\frac{\left(P_{t-s}\{f|\nabla f|\}\right)^{2}}{P_{t-s} f^{2}}\right) \mathrm{d} s \\
& \leq 4 c^{2} \int_{0}^{t} \mathrm{e}^{-2 \lambda(t-s)}\left(P_{t}|\nabla f|^{2}\right) \mathrm{d} s=\frac{2 c^{2}\left(1-\mathrm{e}^{-2 \lambda t}\right)}{\lambda} P_{t}|\nabla f|^{2}, \quad t \geq 0 .
\end{aligned}
$$

Since $\lim _{t \rightarrow \infty} P_{t} g=\mu(g)$ for $g \in \mathscr{B}_{b}(M)$ due to the ergodicity, by letting $t \rightarrow \infty$ we prove the log-Sobolev inequality for (3.4) for $C=\frac{2 c^{2}}{\lambda}$. Indeed, by the local Poincaré inequality and [24, Theorem 3.1], the weak Poincaré inequality

$$
\mu\left(f^{2}\right) \leq \alpha(r) \mu\left(|\nabla f|^{2}\right)+r\|f\|_{\infty}^{2}, \quad r>0, \mu(f)=0
$$

holds for some $\alpha:(0, \infty) \rightarrow(0, \infty)$. By [24, Theorem 2.1], this implies

$$
\lim _{t \rightarrow 0} \sup _{\|g\|_{\infty} \leq 1} \mu\left(\left|P_{t} g-\mu(g)\right|^{2}\right)=0
$$

Proof of Corollary 2.4. We first observe that the proof of [37, Theorem 4.2] works also for the non-symmetric case with $\nabla Z$ in place of $\operatorname{Hess}_{V}$, so that for some constant $c_{1}>0$,

$$
\left\|P_{t}\right\|_{L^{2}(\mu) \rightarrow L^{\infty}(\mu)} \leq \exp \left[c_{1}+\frac{c_{1}}{t}\left(1+\Lambda_{1}^{-1}\left(c_{1} t^{-1}\right)+\Lambda_{2}^{-1}\left(c_{1}^{-1} t\right)\right)\right], \quad t>0 .
$$

Since in the symmetric case we have $\left\|P_{t}\right\|_{L^{1}(\mu) \rightarrow L^{\infty}(\mu)} \leq\left\|P_{t / 2}\right\|_{L^{2}(\mu) \rightarrow L^{\infty}(\mu)}^{2}$, the first assertion follows immediately from Theorem 2.3. 
As for the non-symmetric case, since

$$
\left\|P_{t}\right\|_{L^{1}(\mu) \rightarrow L^{\infty}(\mu)} \leq\left\|P_{t / 2}\right\|_{L^{1}(\mu) \rightarrow L^{2}(\mu)}\left\|P_{t / 2}\right\|_{L^{2}(\mu) \rightarrow L^{\infty}(\mu)},
$$

by Theorem 2.3 and (5.2) it suffices to prove

$$
\left\|P_{t}\right\|_{L^{1}(\mu) \rightarrow L^{2}(\mu)} \leq \exp \left[c^{\prime}+c^{\prime} H\left(c^{\prime} t^{-1}\right)\right], \quad t>0
$$

for some constant $c^{\prime}>0$. According to [25, Theorem 2.1], (5.2) implies the super log-Sobolev inequality (3.3) for

$$
\beta(r):=c+\frac{c}{r}\left\{1+\Lambda_{1}^{-1}\left(c r^{-1}\right)+\Lambda_{2}^{-1}\left(c^{-1} r\right)\right\}, \quad r>0
$$

for some (possibly different) constant $c>0$. Then Proposition 3.3 with $p=1, q=2$ and $\gamma(r):=\frac{\operatorname{trh}(r-1)}{(r-1) \int_{0}^{1} s^{-1} h(s) \mathrm{d} s}$ implies (5.3).

\section{Proofs of Theorems 2.5-2.6}

Proof of Theorems 2.5. Let $X_{t}(x)$ solve (2.17) with initial point $x$. By Itô's formula and condition (2.18) we obtain

$$
\begin{aligned}
\mathrm{d} \mid & X_{t}(x)-\left.X_{t}(y)\right|^{p} \\
\leq & \mathrm{d} M_{t}+p\left|X_{t}(x)-X_{t}(y)\right|^{p-2}\left\{\frac{(p-2) \mid\left(\sigma\left(X_{t}(x)\right)-\left.\sigma\left(X_{t}(y)\right)^{*}\left(X_{t}(x)-X_{t}(y)\right)\right|^{2}\right.}{\left|X_{t}(x)-X_{t}(y)\right|^{2}}\right. \\
& +\left\|\sigma\left(X_{t}(x)\right)-\sigma\left(X_{t}(y)\right)\right\|_{H S}^{2}+\left\langle b\left(X_{t}(x)-b\left(X_{t}(y)\right), X_{t}(x)-X_{t}(y)\right\rangle\right\} \mathrm{d} t \\
\leq & \mathrm{d} M_{t}-p K_{p}\left|X_{t}(x)-X_{t}(y)\right|^{p} \mathrm{~d} t
\end{aligned}
$$

for some martingale $M_{t}$. This implies

$$
\mathbb{E}\left|X_{t}(x)-X_{t}(y)\right|^{p} \leq \mathrm{e}^{-p K_{p} t}|x-y|^{p}, \quad t \geq 0, x, y \in \mathbb{R}^{d},
$$

and thus,

$$
\begin{aligned}
\left|\nabla P_{t} f(x)\right| & \leq \limsup _{y \rightarrow x} \mathbb{E}\left(\frac{\left|f\left(X_{t}(x)\right)-f\left(X_{t}(y)\right)\right|}{\left|X_{t}(x)-X_{t}(y)\right|} \cdot \frac{\left|X_{t}(x)-X_{t}(y)\right|}{|x-y|}\right) \\
& \leq \mathrm{e}^{-K_{p} t}\left(P_{t}|\nabla f|^{\frac{p}{p-1}}\right)^{\frac{p-1}{p}} .
\end{aligned}
$$

Then the desired assertion follows from Proposition 3.1.

Proof of Theorem 2.6. (1) We reformulate (2.17) as

$$
\mathrm{d} X_{t}=b\left(X_{t}\right) \mathrm{d} t+\sqrt{2}\left(\sigma_{0}\left(X_{t}\right) \mathrm{d} B_{t}^{\prime}+\lambda_{0} \mathrm{~d} B_{t}^{\prime \prime}\right),
$$


where $B_{t}^{\prime}$ and $B_{t}^{\prime \prime}$ are independent $d$-dimensional Brownian motions. For any $x \neq y$, let $X_{t}$ solve this SDE with $X_{0}=x$, and let $Y_{t}$ solve the following coupled SDE with $Y_{0}=y$ :

$$
\mathrm{d} Y_{t}=b\left(Y_{t}\right) \mathrm{d} t+\sqrt{2} \sigma_{0}\left(Y_{t}\right) \mathrm{d} B_{t}^{\prime}+\lambda_{0} \sqrt{2}\left(\mathrm{~d} B_{t}^{\prime \prime}-2 \frac{\left\langle X_{t}-Y_{t}, \mathrm{~d} B_{t}^{\prime \prime}\right\rangle\left(X_{t}-Y_{t}\right)}{\left|X_{t}-Y_{t}\right|^{2}}\right) .
$$

That is, under the flat metric we have made coupling by reflection for $B_{t}^{\prime \prime}$ and coupling by parallel displacement for $B_{t}^{\prime}$. Obviously, the coupled SDE has a unique solution up to the coupling time

$$
T_{x, y}:=\inf \left\{t \geq 0: X_{t}=Y_{t}\right\}
$$

We set $Y_{t}=X_{t}$ for $t \geq T_{x, y}$ as usual. Then by (2.19) and Itô's formula, we obtain

$$
\mathrm{d}\left|X_{t}-Y_{t}\right| \leq \mathrm{d} M_{t}+\left\{\left(K_{1}+K_{2}\right) 1_{\left\{\left|X_{t}-Y_{t}\right| \leq r_{0}\right\}}-K_{2}\right\}\left|X_{t}-Y_{t}\right| \mathrm{d} t, \quad t \leq T_{x, y}
$$

for

$$
\mathrm{d} M_{t}:=\frac{\sqrt{2}\left\langle 2 \lambda_{0} \mathrm{~d} B_{t}^{\prime \prime}+\left(\sigma_{0}\left(X_{t}\right)-\sigma_{0}\left(Y_{t}\right)\right) \mathrm{d} B_{t}^{\prime}, X_{t}-Y_{t}\right\rangle}{\left|X_{t}-Y_{t}\right|}
$$

being a martingale with

$$
\mathrm{d}\langle M\rangle_{t} \geq 8 \lambda_{0}^{2} \mathrm{~d} t
$$

By repeating the argument leading to (4.3), it is easy see that (6.3) and (6.4) imply

$$
\mathbb{E}\left|X_{t}-Y_{t}\right| \leq c \mathrm{e}^{-\lambda t}|x-y|, \quad t \geq 0
$$

for some constants $c, \lambda>0$ independent of $x, y$. Therefore,

$$
\left|\nabla P_{t} f\right| \leq c \mathrm{e}^{-\lambda t}\|\nabla f\|_{\infty}, \quad t \geq 0, f \in C_{b}^{1}\left(\mathbb{R}^{d}\right)
$$

so that the first assertion follows from Proposition 3.1.

(2) According to [40, Theorem 1.1], $\sigma \sigma^{*} \geq \lambda_{0}^{2} I$ and (2.21) imply the log-Harnack inequality

$$
P_{t}(\log f)(x) \leq \log P_{t} f(y)+\frac{c_{1}|x-y|^{2}}{1-\mathrm{e}^{-c_{2} t}}, \quad t \geq 0, x, y \in \mathbb{R}^{d}, 0 \leq f \in \mathscr{B}_{b}\left(\mathbb{R}^{d}\right)
$$

for some constants $c_{1}, c_{2}>0$. Next, condition (2.21) implies

$$
\left|\nabla P_{t} f\right|^{2} \leq \mathrm{e}^{2 K t} P_{t}|\nabla f|^{2}
$$

see e.g. [?]RW10. Combining these with the log-Sobolev inequality, we prove the second assertion as in (c) in the proof of Theorem 2.1.

(3) According to the proof of Theorem 2.5, the condition (2.18) implies the gradient estimate (6.1). Next, by Proposition 3.4, the ultracontractivity and (6.1) imply

$$
\left|\nabla P_{t} f\right| \leq c(p) \mathrm{e}^{-\lambda t}\left(P_{t}|\nabla f|^{\frac{p}{p-1}}\right)^{\frac{p-1}{p}}, \quad t \geq 0, f \in C_{b}^{1}\left(\mathbb{R}^{d}\right)
$$

for some $c(p)>0$ and $\lambda>0$ independent of $p$. Then the proof if finished by Proposition 3.1 . 
Acknowledgement. The author would like to thank Jian Wang and the referee for helpful comments and corrections.

\section{References}

[1] S. Aida, T. Masuda, I. Shigekawa, Logarithmic Sobolev inequalities and exponential integrability, J. Funct. Anal. 126(1994), 83-101.

[2] S. Aida, D. Stroock, Moment estimates derived from Poincaré and logarithmic Sobolev inequalities, Math. Res. Lett. 1(1994), 75-86.

[3] M. Arnaudon, A. Thalmaier, F.-Y. Wang, Harnack inequality and heat kernel estimate on manifolds with curvature unbounded below, Bull. Sci. Math. 130(2006), 223-233.

[4] D. Bakry, M. Emery, Hypercontractivité de semi-groupes de diffusion, C. R. Acad. Sci. Paris. Sér. I Math. 299(1984), 775-778.

[5] D. Bakry, I. Gentil, M. Ledoux, Hypercontractivity of Hamilton-Jacobi equations, J. Math. Pures Appl. 80(2001), 669-696.

[6] D. Bakry, I. Gentil, M. Ledoux, Analysis and Geometry of Markov Diffusion Operators, Springer, 2014.

[7] D. Bakry, I. Gentil, M. Ledoux, On Harnack inqualities and optimal transportation, Ann. Sc. Norm. Super. Pisa Cl. Sci. (5) XIV(2015), 705-727.

[8] M.-F. Chen, F.-Y. Wang, Estimates of logarithmic Sobolev constant: an improvement of Bakry-Emery criterion, J. Funct. Anal. 144(1997), 287-300.

[9] M.-F. Chen, F.-Y. Wang, Estimation of spectral gap for elliptic operators, Trans. Amer. Math. Soc. 349(1997), 1239-1267.

[10] E. B. Davies, B. Simons, Ultracontractivity and heat kernel for Schrördinger operators and Dirichlet Laplacians, J. Funct. Anal. 59(1984), 335-395.

[11] A. Eberle, Reflection coupling and Warsserstein contractivity without convecity, C. R. Acad. Sci. Paris. Sér. I Math. 349(2011), 1101-1104.

[12] A. Eberle, Reflection couplings and contraction rates for diffusions, to appear in Probab. Thero. Relat. Fields.

[13] M. Emery, Stochastic Calculus in Manifolds, Springer-Verlag, Berlin, 1989, with an appendix by P.-A. Meyer.

[14] L. Gross, Logarithmic Sobolev inequalities and contractivity properties of semigroups, Lecture Notes in Math. 1563, Springer-Verlag, 1993. 
[15] W. S. Kendall, Nonnegative Ricci curvature and the Brownian coupling property, Stochastics 19(1986), 111-129.

[16] K. Kuwada, Duality on gradient estimates and Wasserstein controls, J. Funct. Anal. 258 (2010), 3758-3774.

[17] K. Kuwada, Gradient estimate for Markov kernels, Wasserstein control and HopfLax formula, In "Potential Theory and Its Related Fields", 61-80, RIMS Kôkyûroku Bessatsu, B43, Res. Inst. Math. Sci. (RIMS), Kyoto, 2013.

[18] D. Luo, J. Wang, Exponential convergence in $L^{p}$-Warsserstein distance for diffusion processes without uniform dissipative drift, to appear in Math. Nachr.

[19] L. Miclo, On hyperboundedness and spectrum of Markov operators, Inven. Math. 200(2015), 311-343.

[20] F. Otto, The geometry of dissipative evolution equations: the porous medium equation, Comm. Part. Diff. Equat. 26(2001), 101-174.

[21] F. Otto, C. Villani, Generalization of an inequality by Talagrand and links with the logarithmic Sobolev inequality, J. Funct. Anal. 173(2000), 361-400.

[22] E. Priola, F.-Y. Wang, Gradient estimates for diffusion semigroups with singular coefficients, J. Funct. Anal. 236(2006), 244-264.

[23] M.-K. von Renesse, K.-T. Sturm, Transport inequalities, gradient estimates, entropy, and Ricci curvature, Comm. Pure Appl. Math. 58(2005), 923-940.

[24] M. Röckner, F.-Y. Wang, Weak Poincaré inequalities and convergence rates of Markov semigroups, J. Funct. Anal. 185(2001), 564-603.

[25] M. Röckner, F.-Y. Wang, Supercontractivity and ultracontractivity for (non-symmetric) diffusion semigroups on manifolds, Forum Math. 15(2003), 893-921.

[26] M. Röckner, F.-Y. Wang, Spectrum for a class of (nonsymmetric) diffusion operators, Bull. London Math. Soc. 36(2004), 95-104.

[27] M. Rockner, F.-Y. Wang, Log-Harnack Inequality for Stochastic differential equations in Hilbert spaces and its consequences, Infinite Dimensional Analysis, Quantum Probability and Related Topics 13(2010), 27-37.

[28] J. Shao, Hamilton-Jacobi semi-groups in infinite dimensional spaces, Bull. Sci. math. 130(2006), 720-738.

[29] W. Stannat, (Nonsymmetric) Dirichlet operators on $L^{1}$ : Existence, uniqueness and associated Markov processes, Ann. Sc. Norm. Super. Pisa Cl. Sci. 28(1999) 99-140.

[30] C. Villani, Topics in Optimal Transportation, Amer. Math. Soc. 2003. 
[31] C. Villani, Optimal Transpot, Old and New, Springer, 2009.

[32] F.-Y. Wang, Application of coupling method to the Neumann eigenvalue problem, Probab. Theory Relat. Fields 98(1994), 299-306.

[33] F.-Y. Wang, Logarithmic Sobolev inequalities on noncompact Riemannian manifolds, Probab. Theory Relat. Fields 109(1997), 417-424.

[34] F.-Y. Wang, Functional inequalities for empty essential spectrum, J. Funct. Anal. 170(2000), 219-245.

[35] F.-Y. Wang, Probability distance inequalities on Riemannian manifolds and path spaces, J. Funct. Anal. 206(2004), 167-190.

[36] F.-Y. Wang, Functional Inequalities, Markov Semigroups and Spectral Theory, Science Press, 2005, Beijing.

[37] F.-Y.Wang, Log-Sobolev inequalities: different roles of Ric and Hess, Ann. Probab. $37(2009), 1587-1604$.

[38] F.-Y.Wang, Log-Sobolev inequality on non-convex manifolds, Adv. Math. 222(2009), $1503-1520$.

[39] F.-Y.Wang, Harnack inequalities on manifolds with boundary and applications, J. Math. Pures Appl. 94(2010) 304-321.

[40] F.-Y. Wang, Harnack inequality for SDE with multiplicative noise and extension to Neumann semigroup on nonconvex manifolds, Ann. Probab. 39(2011), 1449-1467.

[41] F.-Y.Wang, Criteria on spectral gap of Markov operators, J. Funct. Anal. 266(2014), $2137-2152$.

[42] F.-Y. Wang, Analysis for Diffusion Processes on Riamnnian Manifolds, Springer, 2014.

[43] F.-Y. Wang, Asymptotic couplings by reflection and applications for nonlinear monotone SPDEs, Nonlinear Anal. 117(2015), 169-188. 